\title{
Measurement of Harmonic Voltage, Current, and Power at Industrial Frequency
}

\author{
Lu Zuliang \\ National Institute of Metrology \\ P. R. China
}

\section{Introduction}

In recent years, power quality analyzers and revenue meters with harmonic analyzing function have increasingly been used to monitor the state of the power supply network. The precise capability of revenue meters to measure electrical energy under non-sinusoidal conditions has been questioned on the basis of some IEC [IEC, 2003] standards and international recommendations. For EMC, harmonic tests are prescribed in the IEC documents [IEC, 2001, 2002] to ensure that appliances including televisions, computers, lamps, and motors do not produce harmonic currents that exceed allowable limits when they are operated under sinusoidal voltage. The harmonic measurement is an important task too in the background of the smart grid.

Many harmonic analyzers with variable levels and harmonic sources with determined accuracies have been offered in the market even as new instruments are being developed. At the same time, these have created a need for national laboratories to provide calibration facilities and traceability for harmonic quantities, including harmonic voltage, harmonic current, and harmonic power.

To respond to the changing needs of the industry for non-traditional electrical measurements, many national laboratories (NMIs) have developed standards for power measurements under non-sinusoidal waveform conditions. A harmonic power standard equipment was also developed at the National Institute of Metrology of China (NIM) in 2006. This equipment can accurately calibrate harmonic power analyzers and harmonic sources, and calibrate the harmonic-analyzing-function of the revenue meters, including its measuring capability for electrical energy under non-sinusoidal conditions. The uncertainties of the standard equipment are less than $30 \mu \mathrm{V} / \mathrm{V}, 36 \mu \mathrm{A} / \mathrm{A}$, and $42 \mu \mathrm{W} / \mathrm{VA}$ for harmonic voltage, harmonic current, and harmonic power measurements, respectively, at fundamental frequencies of 50 and $60 \mathrm{~Hz}$ and operating ranges of up to $50 \mathrm{~A}, 500 \mathrm{~V}$, and $60^{\text {th }}$ order harmonics.

The harmonic power standard equipment is introduced in this chapter as an example. Its measurement functions for harmonic voltage, harmonic current, and harmonic power at industrial frequency are described, with aspects such as its principle, setup, uncertainty evaluation, and experimental validation also covered. 
The digital sampling technique is widely adopted for commercial harmonic analyzers. For standard equipment, this technique must be developed to a higher level to satisfy calibration requirements. The standard equipment of the NIM is also based on digital sampling techniques. Unlike that proposed in general sampling theory, however, the synchronization between sampling rate and signal frequency is not a stringent requirement for the NIM equipment. The leakage effect that results from such an asynchronous case is compensated for by the introduction of a novel algorithm. The engineering practicality of this algorithm is demonstrated and its calculation is limited to a couple of seconds, without the need for an especially large computing space.

The uncertainty evaluation for the standard equipment is based primarily on experiments. The standard equipment is tested using the national AC voltage and AC current standards at variable frequency points to determine frequency characteristics. Some special factors for harmonic conditions are then considered; these include the small harmonic components, leakage between harmonics, noise, and nonlinear effect of frequency. Finally, some experiments are designed and implemented to validate the uncertainty.

The principles and methods of the algorithm are designed to achieve higher accuracy. The uncertainty evaluation is carried out from the frequency to the harmonic feature. These attributes would be of significant reference for researchers, engineers, and students in developing higher quality commercial instruments or in general study. Based on these principles and methods the readers can improve system and reach a higher accuracy and a better function.

Further investigation to extend the capability of the algorithm for impedance measurement, higher frequency measurement, or other AC measurements would also be valuable.

The equipment system is introduced in Section 2. The algorithm is described in Section 3. The uncertainty of harmonic measurement is conceptually shown in Section 4 . The harmonic voltage and harmonic current, their phase shifts, and harmonic power are discussed in Sections 5, 6, 7, and 8, respectively. The experimental validation is presented in Section 9.

The core materials on which this chapter is based are taken from [Lu et al., 2010], and some concepts in [Lu et al., 2008a, 2008b, 2008c] are extended. A primary form of the algorithm is discussed in [Lu, 1988], and its detailed analysis and application can be found in [Lu, 1991].

\section{System}

A block diagram of the NIM harmonic power standard equipment, including some general hardware, is shown in Fig. 2.1. Two commercial high-accuracy digital sampling voltmeters (DVMs) with type of HP3458A are employed as A/D converters (ADCs) to measure the instantaneous values of voltage and current signals. Non-sinusoidal signals are provided by two commercial programmable signal generators, one with the type of Fluke 6100A and another NST3500 from Chinese manufacturer. A set of resistive dividers have been developed to extend voltage ranges to $8,15,30,60,120,240$, and $500 \mathrm{~V}$ using selected resistors with a low time constant and low temperature coefficient. The resistance values are designed in such a way that the operating current does not introduce a significant heating effect. Another set of resistive dividers provide protection to reduce the effect of stray capacitance. A set of shunts (provided by SP of Sweden) are used to extend current ranges to $0.1,0.2,0.5,1,2,5,10$, and $20 \mathrm{~A}$, while a current transformer is adopted for $50 \mathrm{~A}$. 


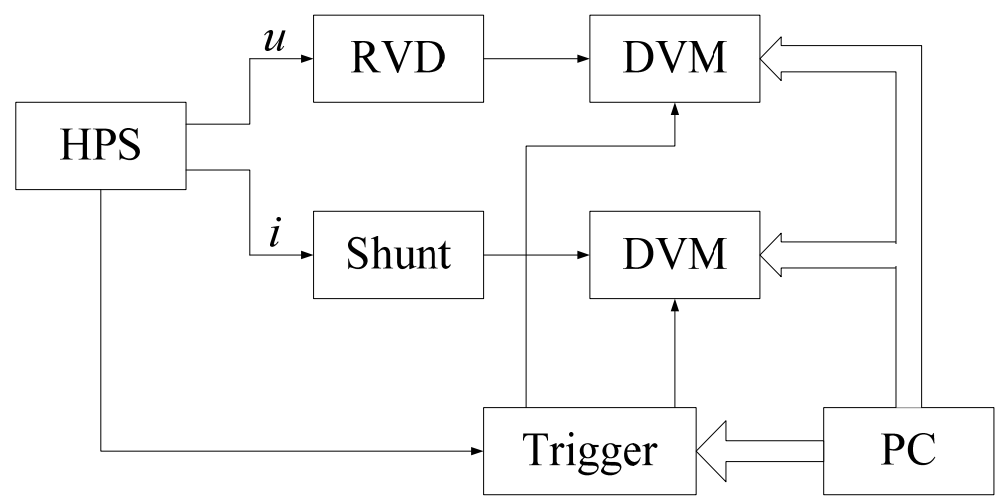

Fig. 2.1. Block Diagram of the harmonic power standard equipment; where HPS is harmonic power source, RVD is resistive voltage divider, and DVM is digital voltage meter.

The full-scale output signals of the voltage dividers and shunts are $0.8 \mathrm{~V}$ regardless of the voltage/current ranges, which are the input signals of the two corresponding DVMs.

A sampling trigger is developed to enable equal interval sampling between two contiguous sampling data, and synchronization sampling between voltage and current signals.

For the practical sampling strategy, 1680 samples over about four signal periods are computed on a PC, in accordance with the algorithm. The calculated results are voltage, current, and power at the DC, fundamental, and every harmonic order up to the $60^{\text {th }}$.

\section{Algorithm}

Generally, power sources and digital sampling meters have their own internal time clocks. Ensuring synchronous sampling capability in both instruments is difficult and often impossible. Such an asynchronous condition generates leakage errors in harmonic analysis because of the noncorrespondence between the accumulated values of the sampling data and the integral values in a precise period.

To resolve this issue, the authors in [Ihlenfeld et al., 2003] developed a method in which one time clock is adopted in the standard equipment, not only for the source (source of standard equipment, SSE), but also for the meter (meter of standard equipment, MSE). When a meter under test (meter under test, MUT) is calibrated, the SSE provides output signals for the MSE and MUT, resulting in synchronous sampling and appropriate calibration results. However, problems occur when calibrating another source (source under test, SUT) that has its own time clock. In such a case, due to two different time clocks are used, MSE calibration under synchronous sampling is difficult to accomplish, consequently resulting in poor calibration.

Non-integer-period sampling (NIPS) resolves this problem [Lu,1991]. It generates good results for both the MUT and SUT, without needing any special time clock and other equipment. The leakage effect persists, but can be overcome by the algorithm developed in this study for more general-purpose applications. The following section describes the algorithm. 


\subsection{Non-integer-period sampling}

A period signal can be written as

$$
y(x)=a_{0}+\sum_{k=1}^{\infty} a_{k} \sin k x+b_{k} \cos k x
$$

where $x=2 \pi t / \tau, \tau$ is the signal period or fundamental period, and $k$ denotes the harmonic order.

All cases can be expressed as

$$
(n+\Delta) h=2 \Pi m,
$$

and the sampling data become

$$
\begin{gathered}
y_{j}=a_{0}+\sum_{k=1}^{\infty} a_{k} \sin j k h+b_{k} \cos j k h \\
j=0,1,2, \ldots, n_{.},
\end{gathered}
$$

where $h$ is the sampling interval, $n$ denotes the number of samples in $m$ periods, and $\Delta$ represents a small quantity with the same means as $n$, noted as a deviation degree of the non-integer-period.

A sampling will be entitled as integer-period sampling (IPS) when $\Delta=0$, and quasi-integerperiod sampling (QIPS) when $|\Delta|<1$. Generally, it will be expressed as $|\Delta h|<\pi$; that is, NIPS.

Fig. 3.1 explains this concept, in which three periods of a signal is divided by 13 samples at the same interval. For case (a), an IPS sample is obtained when 13 samples are computed; for case (b), an NIPS sample is derived when 7 samples are computed; for case (c), a QIPS sample is obtained when 4 samples are calculated.

The key point lies in how many samples are obtained or treated. At any specified uncertainty, a certain IPS sample can always be found for any NIPS sample when the sample number is not limited, so that the NIPS sample is a part of the IPS sample [Lu, 1991].

Similar QIPS samples also exist, as shown in Fig. 3.1. These are the groups of five, eight, and nine samples.

\subsection{Orthogonal check of the trigonometric function in NIPS}

An accumulation operation is used in measuring harmonic quantities from samples of periodic signals. It is denoted as a linear operator/calculator $A$.

A few detailed forms of $A$ were introduced in [Lu, 1988]. These are the trapezoid formula

$$
A y_{j}=\frac{1}{n}\left(0.5 y_{0}+\sum_{j=1}^{n-1} y_{j}+0.5 y_{n}\right) \text {, }
$$


rectangular (Stenbakken's) compensation

$$
A y_{j}=\frac{1}{n+\Delta}\left(\sum_{j=0}^{n-1} y_{j}+\Delta y_{n}\right)
$$

trapezoid compensation

$$
A y_{j}=\frac{1}{n+\Delta}\left[\sum_{j=1}^{n-1} y_{j}+0.5(1+\Delta)\left(y_{0}+y_{n}\right)\right]
$$
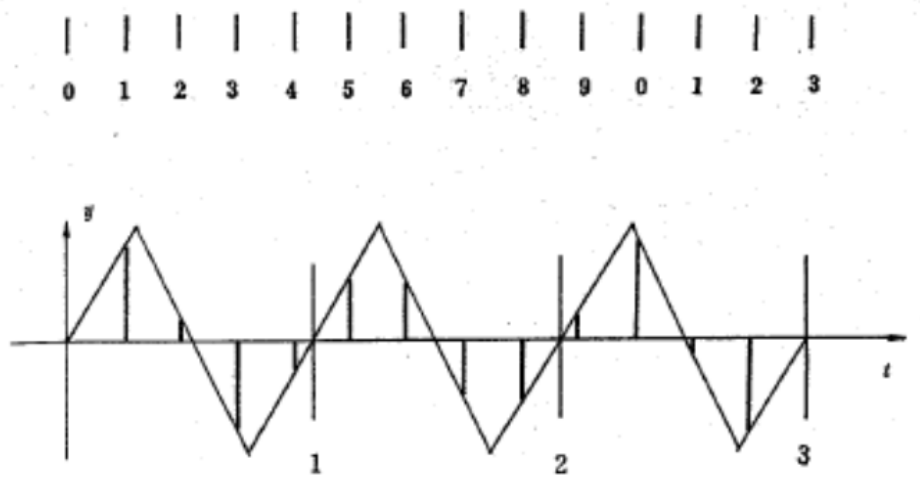

(a)

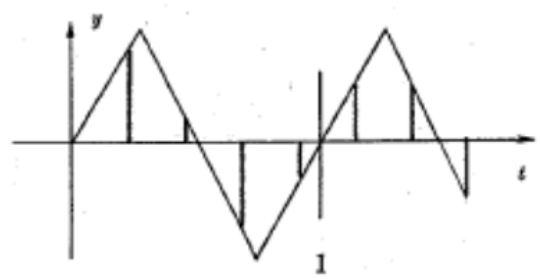

(b)

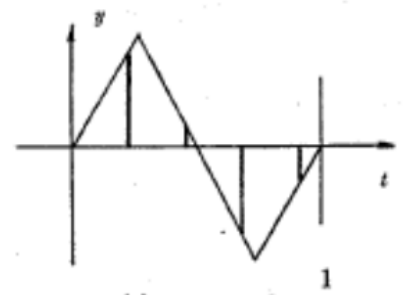

(c)

Fig. 3.1. (a) is an IPS sample, (b) is a NIPS sample, and (c) is a QIPS sample 
In NIPS, the orthogonality of the trigonometric function appears as a deviation.

If

$$
\begin{aligned}
& A \sin j k h=\alpha_{k}, \\
& A \cos j k h=\beta_{k} .
\end{aligned}
$$

Using the characteristic of the trigonometric function proves that

$$
\begin{aligned}
& 2 A(\sin j k h \cos j l h)=\alpha_{k+l}+\alpha_{k-l}, \\
& 2 A(\sin j k h \sin j l h)=-\beta_{k+l}+\beta_{k-l}, \\
& 2 A(\cos j k h \cos j l h)=\beta_{k+l}+\beta_{k-l} .
\end{aligned}
$$

In particular, when $k=0$,

$$
\begin{aligned}
& \alpha_{0}=A \sin j 0 h=A \cdot 0=0, \\
& \beta_{0}=A \cos j 0 h=A \cdot 1=1 .
\end{aligned}
$$

\subsection{Fourier coefficient of the signal in NIPS}

If the Fourier coefficients of the signal described in Eq. (3.1.1) are $\hat{a}_{0}, \hat{a}_{k}, \hat{b}_{k}$, the following hold:

$$
\begin{gathered}
\widehat{a}_{0}=a_{0}+\sum_{k=1}^{\infty} \alpha_{k} a_{k}+\beta_{k} b_{k} \\
\widehat{a}_{k}=2 \alpha_{k} a_{0}+\sum_{l \neq k}^{1, \infty}\left(-\beta_{k+l}+\beta_{k-l}\right) a_{l}+\left(1-\beta_{2 k}\right) a_{k}, \\
+\sum_{l \neq k}^{1, \infty}\left(\alpha_{k+l}+\alpha_{k-l}\right) b_{l}+\alpha_{2 k} b_{k}, \\
\widehat{b}_{k}=2 \beta_{k} a_{0}+\sum_{l \neq k}^{1, \infty}\left(\alpha_{k+l}-\alpha_{k-l}\right) a_{l}+\alpha_{2 k} a_{k}, \\
+\sum_{l \neq k}^{1, \infty}\left(\beta_{k+l}+\beta_{k-l}\right) b_{l}+\left(1+\beta_{2 k}\right) b_{k} .
\end{gathered}
$$

If $w$ is the maximum limit of the harmonic order of the signal shown in Eq. (3.1.1), the Fourier coefficients of the signal can be denoted by a vector a with order $2 w+1$ : 


$$
\mathbf{a}=\left(a_{0}, a_{1}, a_{2}, \cdots, a_{w}, b_{1}, b_{2}, \cdots, b_{w}\right)^{\mathrm{T}} .
$$

Then, its DFT result can be expressed as another vector . $\hat{\mathbf{a}}$ :

$$
\hat{\mathbf{a}}=\left(\hat{a}_{0}, \hat{a}_{1}, \hat{a}_{2}, \cdots, \hat{a}_{w}, \hat{b}_{1}, \hat{b}_{2}, \cdots, \hat{b}_{w}\right)^{T} .
$$

According to Eqs. (3.3.1), (3.3.2), and (3.3.3), we have

$$
\hat{\mathbf{a}}=\mathbf{F}_{\mathrm{R}} \mathbf{a} .
$$

Thus, the results of a can be calculated as follows:

$$
\mathbf{a}=\mathbf{F}_{\mathrm{R}}^{-1} \hat{\mathbf{a}},
$$

where $\mathbf{F}_{\mathbf{R}}$ is a matrix with order $2 w+1$ :

$$
\mathbf{F}_{\mathrm{R}}=\left[\begin{array}{lll}
\mathbf{F}_{11} & \mathbf{F}_{12} & \mathbf{F}_{13} \\
\mathbf{F}_{21} & \mathbf{F}_{22} & \mathbf{F}_{23} \\
\mathbf{F}_{31} & \mathbf{F}_{32} & \mathbf{F}_{33}
\end{array}\right] .
$$

Its detailed form will be dependent on the sampling rate and signal period, and will be independent of the amplitude and phase angle of the signal. That is, matrix $\mathbf{F}_{\mathbf{R}}$ is available for all signals when both the sampling rate and signal period are fixed.

Some interesting and useful characteristics of $\mathbf{F}_{\mathbf{R}}$ are as follows:

$$
\begin{gathered}
\mathbf{F}_{11}=1 \\
\mathbf{F}_{12}=\frac{1}{2}\left(\mathbf{F}_{21}\right)^{T}=\left(a_{1}, a_{2}, \cdots, a_{w}\right) \\
\mathbf{F}_{13}=\frac{1}{2}\left(\mathbf{F}_{31}\right)^{T}=\left(\beta_{1}, \beta_{2}, \cdots, \beta_{w}\right) \\
\left(\mathbf{F}_{22}\right)_{k l}=\left(-\beta_{k+l}+\beta_{k-l}\right) \\
\left(\mathbf{F}_{23}\right)_{k l}=\left(a_{k+l}+a_{k-l}\right) \\
\left(\mathbf{F}_{32}\right)_{k l}=\left(a_{k+l}-a_{k-l}\right) \\
\left(\mathbf{F}_{33}\right)_{k l}=\left(\beta_{k+l}+\beta_{k-l}\right)
\end{gathered}
$$

where $k, l=1,2$, to $w$.

When $w=3$, the matrix of $\mathbf{F}_{\mathbf{R}}$ can be constructed as 


$\begin{array}{ccccccc}1 & \alpha_{1} & \alpha_{2} & \alpha_{3} & \beta_{1} & \beta_{2} & \beta_{3} \\ 2 \alpha_{1} & 1-\beta_{2} & \beta_{1}-\beta_{3} & \beta_{2}-\beta_{4} & \alpha_{2} & \alpha_{3}-\alpha_{1} & \alpha_{4}-\alpha_{2} \\ 2 \alpha_{2} & \beta_{1}-\beta_{3} & 1-\beta_{4} & \beta_{1}-\beta_{5} & \alpha_{3}+\alpha_{1} & \alpha_{4} & \alpha_{5}-\alpha_{1} \\ 2 \alpha_{3} & \beta_{2}-\beta_{4} & \beta_{1}-\beta_{5} & 1-\beta_{6} & \alpha_{4}+\alpha_{2} & \alpha_{5}+\alpha_{1} & \alpha_{6} \\ 2 \beta_{1} & \alpha_{2} & \alpha_{3}+\alpha_{1} & \alpha_{4}+\alpha_{2} & 1+\beta_{2} & \beta_{1}+\beta_{3} & \beta_{2}+\beta_{4} \\ 2 \beta_{2} & \alpha_{3}-\alpha_{1} & \alpha_{4} & \alpha_{5}+\alpha_{1} & \beta_{1}+\beta_{3} & 1+\beta_{4} & \beta_{1}+\beta_{5} \\ 2 \beta_{3} & \alpha_{4}-\alpha_{2} & \alpha_{5}-\alpha_{1} & \alpha_{6} & \beta_{2}+\beta_{4} & \beta_{1}+\beta_{5} & 1+\beta_{6}\end{array}$

\subsection{Determination of the period}

In the algorithm, the period must first be determined. For the standard equipment, the signal period is cursorily known. A more accurate result can be calculated using $\Delta$.

The period end point can be assumed located between points $y_{n}$ and $y_{n+1}$. Its value is exactly $y_{0}$. The period exists in the relationship of $\left(y_{n}, y_{0}, y_{n+1}\right)$. The quantity is calculated [Lu, 1986] as

$$
\Delta=\left(y_{0}-y_{\mathrm{n}}\right) /\left(-y_{\mathrm{n}}+y_{\mathrm{n}+1}\right) .
$$

The other similar relationships of $\left(y_{0}, y_{n+1}, y_{1}\right)$ can also be used. A more precise formula is [Zhang J.Q., 1996]

$$
\Delta=\left(y_{0}+y_{1}-y_{n}-y_{n+1}\right) /\left(-y_{0}+y_{1}-y_{n}+y_{n+1}\right) .
$$

The selection of the starting point of $y_{0}$ is important; it must be located in a region with a large slope. Under sinusoidal conditions, for example, it should be a zero-crossing point, or a point with a minimum value among sampling data.

Other approaches to determining the period are also available. For example, the relationships of the calculated values of phase angles as (5.1.5) about the first period and the second period [Dai, 1989] can be used.

\subsection{Practical implementation of DFT and compensation}

For input sampling data, the practical implementing process of DFT is as follows:

1. to determine $\Delta$ according to Eq. (3.4.1) or (3.4.2);

2. to calculate the sampling/discrete interval of $h$ according to Eq. (3.1.2)

3. to calculate the base functions of $\sin j k h$ and $\cos j k h$ which are needed by DFT;

4. $\quad$ to implement DFT with the trapezoid compensation calculator $\boldsymbol{A}$ in Eq.(3.2.3).

Where an important point is that the base functions of $\sin j k h$ and $\cos j k h$ needed by DFT come from the practical case including the signal period and the sampling rate, but not come only from the sampling rate of ADC.

When application of Eq. (3.2.3) in DFT, the term of $y_{j}$ shall be replaced by $y_{j} \sin j k h$ or $y_{j} \cos j k h, j=0,1,2, \cdots n$. 
After this DFT a further compensation will be carried out for higher accuracy. For this aim, in the fact, the eqn. (3.3.7) is recognized by many researchers, but its calculation is very difficult. A large-capacity RAM and long computing time are required to calculate $\mathbf{F}_{\mathrm{R}^{-1}}$ because of the huge matrix. Its $2 w+1$ order, for example, is 121 when $w=60$ in the NIM standard equipment.

For practical implementation, $\mathbf{F}_{\mathrm{R}}$ must be simplified.

As mentioned in Section 3.3, only two types of quantities, $a_{k}$ and $\beta_{k}$, are necessary for all the elements of the matrix. They should be expressed in analyzable forms. According to the expression of $a_{k}$ and $\beta_{k}$ in Eqs. (3.2.4) and (3.2.5), as well as the expression of trapezoid compensation operator $A$ in Eq. (3.2.3), the quantities can be analyzed and described as

$$
\begin{gathered}
\alpha_{k}=\frac{1}{n+\Delta}\left(\sin ^{2} \frac{\Delta k h}{2} \operatorname{ctg} \frac{k h}{2}-\frac{\Delta}{2} \sin \Delta k h\right), \\
\beta_{k}=\frac{1}{n+\Delta}\left(-\frac{1}{2} \sin \Delta k h \operatorname{ctg} \frac{k h}{2}+\Delta \cos ^{2} \frac{\Delta k h}{2}\right) .
\end{gathered}
$$

However, $\beta_{k}=1, a_{k}=0$ when $k=0$. Alternatively, $a_{-k}=-a_{k}, \beta_{-k}=\beta_{k}$.

Some special controls are adopted in the NIM standard equipment to let $\|\mathbf{X}\|<<1$, where $\mathbf{F}_{\mathbf{R}}$ $=\mathbf{I}+\mathbf{X}$ and $\mathbf{I}$ is a unit matrix. Thus, $\mathbf{F}_{\mathbf{R}}{ }^{-1} \approx \mathbf{I}-\mathbf{X}$, where $\mathbf{X}$ can be directly provided according to Eqs. (3.3.6), (3.5.1), and (3.5.2). The calculation of $\mathbf{F}_{\mathbf{R}}{ }^{-1}$ becomes a very easy process. The author notes here that it will be not necessary for the hardware control in principle.

The precision result, a, is directly calculated without any intermediate process. A complete calculation that includes DFT and compensation can be completed within a couple of seconds, without the necessity for an especially large computing space.

The compensation results are dependent on the ratio of the computational error/uncertainty of $\Delta$ to $n$ [Lu, 2008d].

\subsection{Simulation and the effect of noise}

A simulation test is designed to verify the effect of the algorithm and results, as evaluated against those derived from the general DFT method with no compensation. In the general method the sampling interval is regarded as $2 \Pi m / n$, but in the compensation method used in this study, it is $2 \Pi m /(n+\Delta)$, as shown in Eq. (3.1.2). However in this DFT this $\Delta$ value is asked to calculated from the sampling data.

The sampling data from a sinusoidal signal with an amplitude of $0.8 \mathrm{~V}$ (to check for potential computational errors) are simulated on a computer with same interval of $2 \Pi m /(n+\Delta)$ (it becomes a NIPS or QIPS precisenly). The sampling rate is set at 60 sampling data in one period with different $\Delta$ values. The general DFT and proposed algorithm are then used to handle the same sampling data. Their computing values for signal amplitude are provided and then compared with the set value $(0.8 \mathrm{~V})$ to reveal the relative errors (Table 3.6.1). 


\begin{tabular}{|c|c|c|}
\hline \multirow{2}{*}{$\Delta$} & \multicolumn{2}{|c|}{ Rel. error $/(\mu \mathrm{V} / \mathrm{V})$} \\
\cline { 2 - 3 } & General DFT & $\begin{array}{c}\text { Proposed } \\
\text { algorithm }\end{array}$ \\
\hline 0.5 & 3905 & 10.0 \\
\hline 0.2 & 1626 & 1.9 \\
\hline 0.1 & 822 & 0.8 \\
\hline 0.05 & 413 & 0.4 \\
\hline 0.02 & 166 & 0.1 \\
\hline 0.01 & 83 & 0.1 \\
\hline
\end{tabular}

Table 3.6.1. Simulation results for amplitude, $y=0.8 \sin \omega t(\mathrm{~V}), n=60$

The results show that when $n=60$ and $\Delta$ is controlled within 0.05 , the magnitude of the compensation effect can be as much as 1000 times. The relative error reaches the $10^{-7}$ level.

A similar simulation is conducted for the phase difference between two signals with the same sampling rate, but the phase difference is set at $60^{\circ}$. The results are shown in Table 3.6.2.

\begin{tabular}{|c|c|c|}
\hline \multirow{2}{*}{$\Delta$} & \multicolumn{2}{|c|}{ Error/ $\mu \mathrm{rad}$} \\
\cline { 2 - 3 } & General DFT & Proposed algorithm \\
\hline 0.5 & -3714 & -13 \\
\hline 0.2 & -1615 & -2.7 \\
\hline 0.1 & -828 & -1.1 \\
\hline 0.05 & -420 & -0.5 \\
\hline 0.02 & -169 & -0.2 \\
\hline 0.01 & -85 & -0.1 \\
\hline
\end{tabular}

Table 3.6.2. Simulation results for phase difference, $n=60$

On the basis of this analysis, we design a practical sampling method in which 1680 samples over about 4 periods and a $\Delta$ value controlled within 0.04 is applied in the equipment.

For a fixed $\Delta$, the phase-angle values in units of rad are different in view of varied harmonic components. At the fundamental, if the value is $2 \pi m \Delta$, but at the $k$-th harmonics, it is $2 \pi k m \Delta$. Therefore, the same $\Delta$ for higher harmonics produces different errors.

In simulating this case, the same sampling point construction is used for all the harmonics of the non-sinusoidal signal. This approach, which we call "harmonic discrete division," is the foundation of the investigation on harmonic sampling measurement.

This method is adopted to investigate the performance of the algorithm for harmonics. A practical condition is considered; i.e., 1680 samplers (with $\Delta$ ) over 4 fundamental periods. When the $10^{\text {th }}$ order harmonics is simulated, the 1680 points are constructed over 40 sinusoidal waveforms. The results are shown in Table 3.6.3. 


\begin{tabular}{|l|l|l|l|l|l|l|l|}
\hline Order & 1 & 10 & 20 & 30 & 40 & 50 & 60 \\
\hline Error/ $(\mathrm{nV} / \mathrm{V})$ & 0.2 & 21 & 68 & 102 & 74 & 55 & 298 \\
\hline
\end{tabular}

Table 3.6.3. Amplitude error after compensation for harmonic components, $y=0.8 \sin \omega t(\mathrm{~V})$ for every harmonic, $\Delta=0.04, n=1680$

The errors that occur after compensation reaches the 10-10 level for the fundamental, and are less than $3 \times 10^{-7}$ up to the $60^{\text {th }}$ order harmonics. This result indicates that the leakage caused by the non-integer-period has been resolved.

Many other tests, including some complex waveforms, are conducted. A characterizing waveform signal discussed below is tested, in which all harmonic components from DC to 60 th order harmonic exist with a relative high percent of amplitude, the largest error is $0.2 \times$ $10^{-6}$. This result can be disregarded for the final estimated uncertainty.

The practical specifications are determined in experiments, such as the calibration traceable to the primary standard of the AC voltage and AC current of the NIM. In such cases, the noise becomes the primary determining factor for uncertainties.

The experimental results show that the noise effect for sinusoidal signal is about (0.5 to 2.0) $\times 10^{-6}$, as validated by Monte Carlo test results [Xue, 2011]. This means that the error from the algorithm (less than $1.0 \times 10^{-6}$ ) is smaller than the effect of noise, and additional efforts to derive higher level algorithm results are not necessary.

\section{Concept for the uncertainty of harmonic measurement}

\subsection{Fiducial error and fiducial uncertainty}

Relative error and relative uncertainty are two concepts often used in calibrations for general quantities. These concepts have also been used in many expressions of harmonic measurement. However, the example given in this study illustrates the need to revisit these concepts.

A harmonic analyzer is used to measure a non-sinusoidal voltage signal with a fundamental $100 \mathrm{~V}$ and a $5^{\text {th }}$ order harmonic $10 \mathrm{~V}$. It measures the $5^{\text {th }}$ order harmonics as $10.0101 \mathrm{~V}$, of which $10 \mathrm{mV}$ is a leakage error from the fundamental $(100 \mathrm{~V})$, whereas $0.1 \mathrm{mV}$ is an error only from the $5^{\text {th }}$ order harmonic $(10 \mathrm{~V})$. In this case, the relative measurement error of the $5^{\text {th }}$ order harmonic of the analyzer is $10.1 \mathrm{mV} / 10 \mathrm{~V}=0.1 \%$.

In another measurement, the analyzer measures a signal with $100 \mathrm{~V}$ of the fundamental plus $0.1 \mathrm{~V}$ of the $5^{\text {th }}$ order harmonic. In this case, the leakage error from the fundamental $(10 \mathrm{mV})$ persists because of the fact that the $100 \mathrm{~V}$ fundamental is unchanged, but the error that comes only from the $5^{\text {th }}$ order harmonic may be $1 \mu \mathrm{V}$. Hence, the relative measurement error is $10 \mathrm{mV} / 0.1 \mathrm{~V}=10 \%$, and not $0.1 \%$ as in the first case.

The example illustrates that the specifications of an analyzer are dependent on the magnitude of the signal to be measured for the relative error concept. Some error components may come from the fundamental. In such a case, the harmonic relative error of the analyzer is variable when the harmonic amplitude varies. This attribute does not correspond with that indicated in the typical error concept. 
The fundamental is the main component in a general non-sinusoidal signal, whereas the harmonics are secondary components. Therefore, the selection of the measurement range in characterizing harmonic analyzers is dependent/determined primarily by the value of the fundamental component.

However, the concept of total harmonic distortion (THD) is related only to the fundamental component or the total RMS value of the harmonics including the fundamental, and not to the individual harmonic component itself.

If the fundamental component is used as reference, then the error is the same in both cases, as shown in the earlier example. That is, $10 \mathrm{mV} / 100 \mathrm{~V}=0.01 \%$. However, we introduce a new error concept called the fiducial harmonic error, developed by the International Vocabulary of Metrology (VIM, Second edition) [BIPM et al., 1993a] (regrettably, the term is not embodied in the Third edition, JCGM 200:2008). This concept is defined as follows:

$$
\delta V_{k}=\Delta V_{k} / V_{1},
$$

where $\Delta V_{k}$ is the absolute error of the $k$-th harmonic, and $V_{1}$ is value of the fundamental.

The harmonic uncertainty concept is expanded with a relative uncertainty concept, $u$ $\left(\Delta V_{k} / V_{k}\right)$, when the quantity itself, $V_{k}$, is taken as the reference and the absolute uncertainty is defined as $u\left(\Delta V_{k}\right)$ :

$$
u\left(\Delta V_{k} / V_{k}\right)=\left[u\left(\Delta V_{k}\right)\right] / V_{k} .
$$

Changing the reference from $V_{k}$ into $V_{1}$, we introduce a new harmonic uncertainty concept and denote it as $u\left(\Delta V_{k} / V_{1}\right)$ :

$$
u\left(\Delta V_{k} / V_{1}\right)=\left[u\left(\Delta V_{k}\right)\right] / V_{1} .
$$

This harmonic uncertainty is defined as the fiducial harmonic uncertainty.

The relationship between the fiducial and relative harmonic uncertainty is

$$
u\left(\Delta V_{k} / V_{1}\right)=u\left(\Delta V_{k} / V_{k}\right) \times\left(V_{k} / V_{1}\right) .
$$

The fiducial harmonic uncertainty is a reasonable choice in expressing harmonic measurement uncertainties, as explained previously.

\subsection{Characterizing waveform signal}

In Eq. (4.1.4), the ratio of $V_{k} / V_{1}$ is an indeterminate variable. To estimate the uncertainty of the equipment, this ratio should be a fixed value to cover all the possible calibration cases. The IEC documents, international recommendations for electricity meters, and EMC provide some typical waveforms [IEC, 2001, 2002, 2003] that can be used to estimate these fixed ratio values. The National Research Council of Canada (NRC) proposed eight different reference waveforms based on actual field-recorded distorted waveforms that can be used for same aim [Arseneau et al., 1995a].

In conclusion, after considering all the different potential test waveforms that can be used, some rules can be assumed as follows: 


$$
\begin{gathered}
V_{0} / V_{1} \leq 0.5, \\
V_{k} / V_{1} \leq 1, \text { when } 2 \leq k \leq 10, \\
V_{k} / V_{1} \leq r / k, \text { when } 10<k \leq 60,
\end{gathered}
$$

where $V_{k}$ is the amplitude of the $k$-th harmonic component, $V_{0}$ denotes the DC component, $V_{1}$ represents the fundamental, and $\gamma$ is a ratio factor.

A characterizing waveform signal, determined according to the aforementioned limit values, is proposed (Table 4.2.1). Its corresponding waveform is shown in Fig 4.2.1. This is only a theoretical signal $(T H D=280 \%)$, conjured to estimate the uncertainty of the harmonic power standard equipment. The signal covers all the possible cases under calibration, so that the harmonic uncertainty claim has universality. It is frequently used for the general analysis and digital evaluation of uncertainty for the harmonic power standard of the NIM [Lu, 2010].

\begin{tabular}{|c|c|c|c|c|c|c|c|c|}
\hline Order & $Y$ & Ratio & Order & $\gamma$ & Ratio & Order & $Y$ & Ratio \\
\hline 0 & & 0.500 & 21 & 3 & 0.143 & 41 & 1 & 0.024 \\
\hline 1 & & 1.000 & 22 & 3 & 0.136 & 42 & 1 & 0.024 \\
\hline 2 & & 0.900 & 23 & 3 & 0.130 & 43 & 1 & 0.023 \\
\hline 3 & & 0.900 & 24 & 3 & 0.125 & 44 & 1 & 0.023 \\
\hline 4 & & 0.900 & 25 & 3 & 0.120 & 45 & 1 & 0.022 \\
\hline 5 & & 0.900 & 26 & 3 & 0.115 & 46 & 1 & 0.022 \\
\hline 6 & & 0.800 & 27 & 3 & 0.111 & 47 & 1 & 0.021 \\
\hline 7 & & 0.800 & 28 & 3 & 0.107 & 48 & 1 & 0.021 \\
\hline 8 & & 0.800 & 29 & 3 & 0.103 & 49 & 1 & 0.020 \\
\hline 9 & & 0.800 & 30 & 3 & 0.100 & 50 & 1 & 0.020 \\
\hline 10 & & 0.750 & 31 & 2 & 0.065 & 51 & 1 & 0.020 \\
\hline 11 & 8 & 0.727 & 32 & 2 & 0.063 & 52 & 1 & 0.019 \\
\hline 12 & 7 & 0.583 & 33 & 2 & 0.061 & 53 & 1 & 0.019 \\
\hline 13 & 6 & 0.462 & 34 & 2 & 0.059 & 54 & 1 & 0.019 \\
\hline 14 & 5 & 0.357 & 35 & 2 & 0.057 & 55 & 1 & 0.018 \\
\hline 15 & 4 & 0.267 & 36 & 2 & 0.056 & 56 & 1 & 0.018 \\
\hline 16 & 3 & 0.188 & 37 & 2 & 0.054 & 57 & 1 & 0.018 \\
\hline 17 & 3 & 0.176 & 38 & 2 & 0.053 & 58 & 1 & 0.017 \\
\hline 18 & 3 & 0.167 & 39 & 2 & 0.051 & 59 & 1 & 0.017 \\
\hline 19 & 3 & 0.158 & 40 & 2 & 0.050 & 60 & 1 & 0.017 \\
\hline 20 & 3 & 0.150 & & & & & & \\
\hline
\end{tabular}

Table 4.2.1. Amplitude ratio of the characterizing signal, $V_{k} / V_{1}$ 


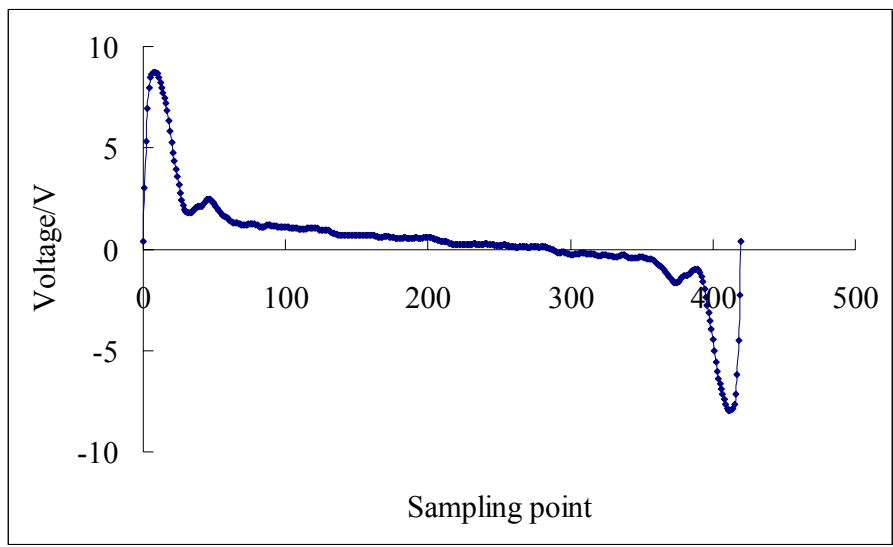

Fig. 4.2.1. Waveform of the characterizing signal.

\section{Traceability of harmonic voltage and its uncertainty}

\subsection{Determination of harmonic voltage}

When Eq. (3.1.1) expresses a non-sinusoidal voltage signal and Eq. (3.1.3) denotes the sampling data under the sampling model of Eq. (3.1.2), the DC voltage, fundamental voltage, and harmonic voltage components can be obtained after calculation according to Eq. (3.3.7). These are expressed as follows:

$$
\begin{gathered}
V_{0}=a_{0}, \\
V_{1}=\sqrt{a_{1}^{2}+b_{1}^{2}}, \\
\varphi_{V 1}=\arctan \left(b_{1} / a_{1}\right), \\
V_{k}=\sqrt{a_{k}^{2}+b_{k}^{2}}, \\
\varphi_{V k}=\arctan \left(b_{k} / a_{k}\right),
\end{gathered}
$$

where $\varphi_{V 1}, \varphi_{V k}$ are the phase angles (against a reference time point) of the fundamental and harmonics, respectively, and $k=2,3, \ldots w$.

The experimental determination of the technical specifications of the NIM harmonic power standard equipment is discussed next, beginning with harmonic voltage.

\subsection{Frequency characteristic}

The frequency characteristic of the standard equipment can be initially determined by adopting the existing National AC Voltage Primary Standard for thermo-converters. In this process, the stable sinusoidal signals are exported at the selected frequency points that correspond to the fundamental, $10^{\text {th }}, 20^{\text {th }}, 30^{\text {th }}, 40^{\text {th }}, 50^{\text {th }}$, and $60^{\text {th }}$ orders. Their amplitudes 
are set to correspond to the full range. Such signals are connected in parallel to the AC Voltage Standard and the harmonic power standard equipment (DVM or/and RVD). The experimental results show the differences than the AC Voltage Standard and its uncertainties.

This frequency characteristic is a special frequency attribute. The principal difference is that in the sampling measurement for high frequencies, harmonic discrete division is adopted (see Section 3.6). A similar case exists for the current discussed in Section 5.1 and the phase shift mentioned in Section 7.4, but this is not discussed for simplification.

\subsubsection{Digital Voltage Meter}

Two DVMs (one for voltage, and another for current) are tested. The input range of $0.8 \mathrm{~V}$ is set as the test voltage. The DVMs are self-calibrated in advance through short input operation, and are connected to the reference voltage.

The results indicate that both DVMs have similar characteristics. The relative differences/errors $\left(\Delta V_{k} / V_{k}\right)$ are

less than $1 \mu \mathrm{V} / \mathrm{V}$ at the fundamental,

less than $10 \mu \mathrm{V} / \mathrm{V}$ within the $50^{\text {th }}$ order,

less than $20 \mu \mathrm{V} / \mathrm{V}$ at the $60^{\text {th }}$ order.

Their standard deviations are all less than $3 \mu \mathrm{V} / \mathrm{V}$.

\subsubsection{Resistive Voltage Divider}

In this experiment, test voltages of 50,100, 200, and $500 \mathrm{~V}$ are set. The resistive voltage dividers and buffers are tested together with the DVM. The relative differences/errors $(\Delta$ $V_{k} / V_{k}$ ) in the results are not considered for correction, but are taken as a part of uncertainty according to GUM [BIPM et al., 1995b], so that relevant correction will not be necessary in future simplified practical operations. After evaluating these results and their standard deviations, the uncertainties of the resistive voltage divider (RVD) are obtained (Table 5.2.1).

\begin{tabular}{|l|l|l|l|l|l|l|l|}
\hline Order & 1 & 10 & 20 & 30 & 40 & 50 & 60 \\
\hline $50 \mathrm{~V}$ & 5.22 & 8.05 & 7.36 & 5.25 & 12.6 & 14.4 & 36.3 \\
\hline $100 \mathrm{~V}$ & 8.45 & 9.95 & 10.6 & 10.0 & 8.83 & 6.58 & 35.6 \\
\hline $200 \mathrm{~V}$ & 7.68 & 8.53 & 7.86 & 10.8 & 15.1 & 19.2 & 47.6 \\
\hline $500 \mathrm{~V}$ & 9.50 & 8.81 & 12.6 & 19.6 & 29.2 & 26.7 & 64.2 \\
\hline
\end{tabular}

Table 5.2.1. Relative uncertainties of the voltage without correction, $u\left(\Delta V_{k} / V_{k}\right) /(\mu \mathrm{V} / \mathrm{V})$ $(k=1)$

\subsection{Harmonic characteristic}

The frequency characteristic of the standard equipment obtained in the section above is not the harmonic characteristic required by harmonic measurement. Some other factors that focus on the fiducial uncertainty in Eq. (4.1.3) are considered. 


\subsubsection{Effect of the small component}

The harmonic components are smaller in practical non-sinusoidal signals. The uncertainty estimation at full range in Table 5.2.1 cannot be directly applied to smaller amplitudes. A general method for non-linear amplitudes can be employed, where the ratio of $\left(V_{k} / V_{1}\right)$ is replaced by $\left[0.8\left(V_{k} / V_{1}\right)+0.2\right]$, in which a minimum value of 0.2 (or $\left.20 \%\right)$ is maintained even if $V_{k}=0$. The ratio $0.8: 0.2$ (or $80 \%: 20 \%$ ) is taken from the experimental results. Eq. (4.1.4) then becomes

$$
u\left(\Delta V_{k} / V_{1}\right)=\left[0.8\left(V_{k} / V_{1)}\right)+0.2\right] \times u\left(\Delta V_{k} / V_{k}\right)
$$

\subsubsection{Leakage and noise}

When a frequency signal (for example, a sinusoidal signal corresponding to the $10^{\text {th }}$ order harmonics) is tested in the single tone experiment, other components (from the DC to the $60^{\text {th }}$ order) aside from the input signal itself appear in the DFT computational results. These components should be zero but are non-zero in practice possibly because the output of the source is not a pure single tone but with side bands; this result may also be attributed to both leakage due to faulty compensation and noise.

To overcome the side bands of the source, 3 sets of data $\left(7^{\text {th }}, 8^{\text {th }}, 9^{\text {th }}\right)$ ahead of the input signal $\left(10^{\text {th }}\right)$ and 3 sets of data behind $\left(11^{\text {th }}, 12^{\text {th }}, 13^{\text {th }}\right)$ that same signal in the DFT computational results are excluded in the analysis below.

The rest of the data reflect mainly leakage and noise. An "enlargement test" is designed and implemented to distinguish between both parts. The effect of the non-integer-period is enlarged 10 times ( $\Delta$ is enlarged from 0.04 to 0.4 ). The difference between the two groups of data is regarded as the influence from leakage only, and the remainder is regarded as noise.

The error of the $k$-th harmonic includes all leakages from the DC to the $60^{\text {th }}$ order aside from itself. Therefore, all the contributions from the other harmonics are accumulated into the uncertainty of the $k$-th harmonic.

The contribution of the leakage from the experimental results is $2.5 \mu \mathrm{V} / \mathrm{V}$, and the noise dependent on frequency is $6.0 \mu \mathrm{V} /(k=1)$.

\subsubsection{Non-linearity effect}

When two sinusoidal signals are simultaneously input, such as the $1^{\text {st }}$ and $5^{\text {th }}$ orders, and when the input circuit of the meter (or the output circuit of the source) exhibits non-linearity of frequencies, two new sinusoidal signals, the $4^{\text {th }}$ and $6^{\text {th }}$ orders, appear in the results. This effect occurs too in the single tone experiment.

We design and implement an experiment, in which a signal with the fundamental and $5^{\text {th }}$ order is measured. Another signal with only the $5^{\text {th }}$ order is measured. The difference between the two above-mentioned DFT results at the $4^{\text {th }}$ and $6^{\text {th }}$ orders reflects this nonlinearity. According to the experimental results, the contribution of this effect is $2.2 \mu \mathrm{V} / \mathrm{V}$ $(k=1)$, including other possible influences from the signal source. 


\subsection{Conclusion}

The small component effect of Eq. (5.3.1) is a part of the uncertainty. This component is called the traceability component because of its dependence on the voltage unit.

The leakage, noise, and non-linearity are other parts, but they are independent of the voltage unit. They can be regarded as the resolution component, expressed as SQR $\left(2.5^{2}\right.$ $\left.+6.0^{2}+2.2^{2}\right)=6.85 \mu \mathrm{V} / \mathrm{V}(k=1)$ on the basis of the results in Section 5.3.

Considering the two components (traceability and resolution), the fiducial uncertainty of Eq. (5.3.1) becomes

$$
u_{\mathrm{c}^{2}}\left(\Delta V_{k} / V_{1}\right)=\left[0.8\left(V_{k} / V_{1}\right)+0.2\right]^{2} u^{2}\left(\Delta V_{k} / V_{k}\right)+6.85^{2},
$$

where the parameters of $u\left(\Delta V_{k} / V_{k}\right)$ can be introduced from Table 5.2.1, and the ratio of $\left(V_{k} / V_{1}\right)$ can be adopted from the characterizing signal in Table 4.2.1. The final uncertainties are calculated and shown in Table 5.4.1.

\begin{tabular}{|l|l|l|l|l|l|l|l|}
\hline Order & 1 & 10 & 20 & 30 & 40 & 50 & 60 \\
\hline $50 \mathrm{~V}$ & 8.6 & 9.4 & 7.2 & 7.0 & 7.5 & 7.5 & 10.3 \\
\hline $100 \mathrm{~V}$ & 10.9 & 10.5 & 7.6 & 7.4 & 7.2 & 7.0 & 10.2 \\
\hline $200 \mathrm{~V}$ & 10.3 & 9.7 & 7.3 & 7.5 & 7.7 & 8.0 & 12.3 \\
\hline $500 \mathrm{~V}$ & 11.7 & 9.8 & 7.9 & 8.8 & 9.8 & 8.9 & 15.3 \\
\hline
\end{tabular}

Table 5.4.1. Fiducial uncertainty under the characterizing signal condition, $u_{\mathrm{c}}\left(\Delta V_{k} / V_{1}\right) /(\mu \mathrm{V} / \mathrm{V})(k=1)$

An estimation of $30 \mu \mathrm{V} / \mathrm{V}(k=2)$ for all the voltage ranges can be obtained. At $100 \mathrm{~V}$, however, this estimation amounts to $20 \mu \mathrm{V} / \mathrm{V}$.

\section{Traceability of harmonic current and its uncertainty}

\subsection{Determination of harmonic current}

When Eq. (3.1.1) expresses a non-sinusoidal current signal, and Eq. (3.1.3) denotes the sampling data under the sampling model of Eq. (3.1.2), after computation according to Eq. (3.3.7), the DC current, fundamental current, and harmonic current components can be obtained thus:

$$
\begin{gathered}
I_{0}=a_{0}, \\
I_{1}=\sqrt{a_{1}^{2}+b_{1}^{2}}, \\
\varphi_{I 1}=\arctan \left(b_{1} / a_{1}\right), \\
I_{k}=\sqrt{a_{k}^{2}+b_{k}^{2}}, \\
\varphi_{I k}=\arctan \left(b_{k} / a_{k}\right),
\end{gathered}
$$


where $\varphi_{I 1}, \varphi_{I k}$ are the phase angles (against a reference time point) of the fundamental and harmonics, respectively, and $k=2,3, \ldots w$.

Similar experiments and computations are carried out for the harmonic current specifications of the NIM harmonic power standard equipment.

\subsection{Frequency characteristic}

The shunts in the equipment are tested. The relative differences/errors are not considered as corrections. The current traceability uncertainty in the full range of every shunt is estimated and shown in Table 6.2.1, including the contribution from the Primary Standards (DC resistance, $\mathrm{AC} / \mathrm{DC}$ transfer, and $\mathrm{DC}$ voltage).

\begin{tabular}{|c|c|c|c|c|c|c|c|}
\hline Order & 1 & 10 & 20 & 30 & 40 & 50 & 60 \\
\hline$\leq 5 \mathrm{~A}$ & 10.6 & 11.8 & 11.8 & 11.7 & 10.3 & 11.3 & 17.0 \\
\hline $\begin{array}{c}10 \mathrm{~A}, \\
20 \mathrm{~A}\end{array}$ & 14.6 & 15.5 & 19.1 & 19.0 & 22.5 & 27.4 & 34.5 \\
\hline
\end{tabular}

Table 6.2.1. Traceability uncertainty in the full range of shunts without correction, $u\left(\Delta I_{k} / I_{k}\right) /(\mu \mathrm{A} / \mathrm{A})(k=1)$

An experiment is designed and performed to check the aforementioned estimation (Fig. 6.2.1). An inductive shunt is developed [Zhang J.T. et al., 2007] to produce two equal currents with very high accuracy. In a general series connection of two resistors, a potential at the middle point causes error. The defect is addressed in this study. The results obtained satisfy the estimation in Table 6.2.1.

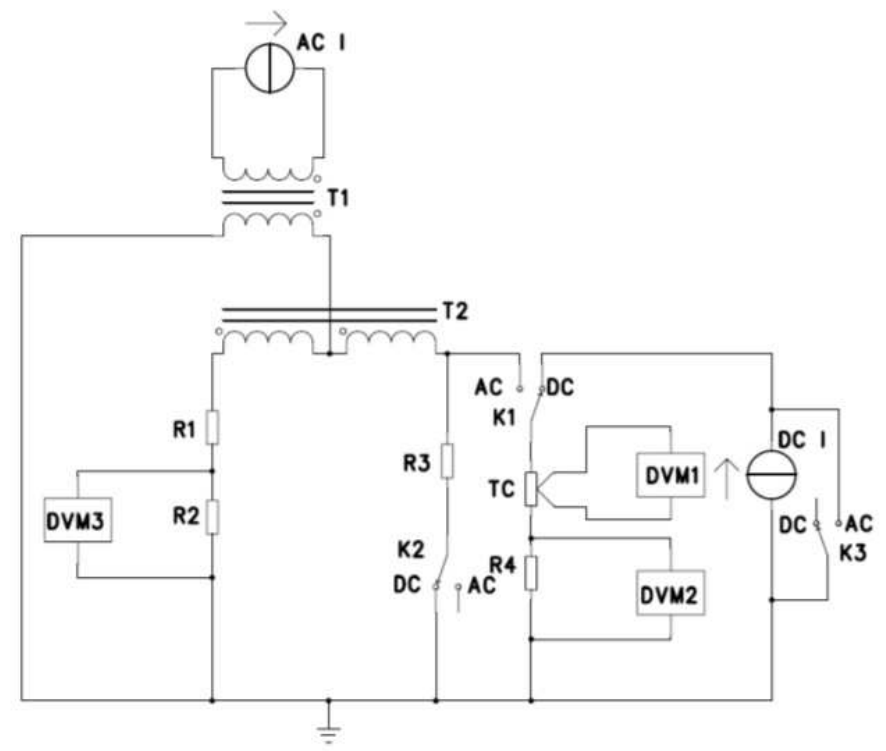

Fig. 6.2.1. Circuit of current traceability. 


\subsection{Harmonic characteristic}

Similar to the voltage case, the fiducial uncertainty of harmonic current $u\left(\Delta I_{k} / I_{1}\right)$ is obtained as

$$
u_{\mathrm{c}}^{2}\left(\Delta I_{k} / I_{1}\right)=u^{2}\left(\Delta I_{k} / I_{1}\right)+7.28^{2},
$$

where

$$
u\left(\Delta I_{k} / I_{1}\right)=\left[0.7\left(I_{k} / I_{1}\right)+0.3\right] u\left(\Delta I_{k} / I_{k}\right)
$$

is the traceability component in which non-linearity of amplitude is considered. For the resolution component, 2.5, 6.0, and $3.3 \mu \mathrm{A} / \mathrm{A}$ are experimentally determined for leakage, noise, and non-linearity of frequencies, respectively.

The digital computational results for the characteristic signal condition are expressed in Table 6.3.1.

\begin{tabular}{|c|c|c|c|c|c|c|c|}
\hline Order & 1 & 10 & 20 & 30 & 40 & 50 & 60 \\
\hline$\leq 5 \mathrm{~A}$ & 14 & 14 & 11 & 11 & 10 & 10 & 11 \\
\hline $\begin{array}{c}10 \mathrm{~A}, \\
20 \mathrm{~A}\end{array}$ & 18 & 16 & 12 & 12 & 12 & 13 & 15 \\
\hline $50 \mathrm{~A}$ & 18 & 16 & 12 & 12 & 12 & 13 & 15 \\
\hline
\end{tabular}

Table 6.3.1. Combined fiducial uncertainty in the characteristic signal, $u_{c}\left(\Delta I_{k} / I_{1}\right) /(\mu \mathrm{A} / \mathrm{A})$ $(k=1)$

The table shows that the experimental results for $50 \mathrm{~A}$ are derived from the current transformer. It also shows that the maximum result is $36 \mu \mathrm{A} / \mathrm{A}(k=2)$, which can be used as an estimation for the harmonic measurement of the standard equipment.

\section{Determination of phase shift and its uncertainty}

The phase difference between voltage and current is an important quantity in power measurement. In harmonic power measurement, every phase difference between the harmonic voltage and harmonic current at the same order from the $2^{\text {nd }}$ to the $60^{\text {th }}$ must be determined. Two problems are discussed: how the phase difference is measured and how its uncertainty is evaluated.

\subsection{Measurement of the phase difference of two signals}

\subsubsection{Phase difference of two voltage signals}

Phase difference can be measured using the sampling approach. The phase difference between two voltages can be calculated using the DFT sampling data results [Lu et al., 2006]:

$$
\varphi_{k}=\arctan \left(b_{2 k} / a_{2 k}\right)-\arctan \left(b_{1 k} / a_{1 k}\right),
$$

where $\varphi_{k}$ is the phase difference of the $k$-th harmonics between voltages $y_{2}$ and $y_{1}$ : 


$$
\begin{aligned}
& y_{1}=a_{10}+\sum_{k=1}^{w}\left(a_{1 k} \sin k \omega t+b_{1 k} \cos k \omega t\right) \\
& y_{2}=a_{20}+\sum_{k=1}^{w}\left(a_{2 k} \sin k \omega t+b_{2 k} \cos k \omega t\right)
\end{aligned}
$$

$a_{1 k}, b_{1 k}\left(a_{2 k}, b_{2 k}\right)$ are the Fourier coefficients of $y_{1}\left(y_{2}\right)$.

The method involves sampling the two voltage signals simultaneously using two ADCs (i.e., DVMs in the standard equipment), and applying DFT to the sampling data and then computing phase difference according to Eq. (7.1.1).

The algorithm introduced in Section 3 can be applied here for precise Fourier coefficients to result in precise phase difference.

Because two different ADCs are used, an intrinsic phase difference occurs, thereby affecting the results. A voltage can be connected parallel to the two channels and the above-mentioned method can be implemented to determine this intrinsic error, which can then be corrected in succeeding measurements [Svensson,1998]. This process can be called producing a $0^{\circ}$ standard.

\subsubsection{Uncertainty of phase difference}

The phase angle definition is included in Eq. (7.1.1); that is,

$$
\varphi=\arctan (b / a) .
$$

Therefore, its uncertainty can be expressed by the amplitude

$$
u^{2}(\varphi)=u^{2}(\arctan (b / a))=\left[b u(a) /\left(a^{2}+b^{2}\right)\right]^{2}+\left[a u(b) /\left(a^{2}+b^{2}\right)\right]^{2} .
$$

If $u(a) \geq u(b)$,

$$
u(\varphi) \leq u(a) / \operatorname{sqrt}\left(a^{2}+b^{2}\right)=u(a) / c,
$$

where $u(a)$ is an absolute uncertainty, whose concept is similar to absolute uncertainty $u\left(\Delta V_{k}\right)$ in Section 4.

In general, $a=(1 \sim 0.71) c \leq c$; thus,

$$
u\left(\varphi_{V k}\right)=(1 \sim 0.71) u\left(\Delta V_{k}\right) / V_{k} \leq u\left(\Delta V_{k}\right) / V_{k}=u\left(\Delta V_{k} / V_{k}\right) .
$$

The uncertainty of phase difference $u\left(\Delta \varphi_{V k}\right)$ between two voltages can be expressed as

$$
u^{2}\left(\Delta \varphi_{V k}\right)=u^{2}\left(\Delta V_{1 k} / V_{1 k}\right)+u^{2}\left(\Delta V_{2 k} / V_{2 k}\right) .
$$

The relative uncertainty of $u\left(\Delta V_{k} / V_{k}\right)$ is discussed in Section 5 for voltage (and Section 6 for current), and determined in experiments. However, some uncertainty factors can be discussed further:

1. Given that the phase angle is dependent on the ratio of Fourier coefficients, the uncertainty of the voltage standard from traceability has no function here.

2. The standard deviation of measurement is an important factor.

3. Leakage and noise are also factors. 
4. The starting point of sampling brings about variation in Fourier coefficients $a$ and $b$. However, the experiment shows that their influence can be disregarded.

5. The compensation of the algorithms is also a primary factor. However, the experiment proves that the residual effect occurring after the compensation using Eq. (3.3.7) is less than the noise. This effect can also be disregarded.

\subsubsection{Phase difference between a voltage signal and a current signal}

Only the shunt in the equipment is discussed in this section. The input signal is a current and the output signal is a voltage, shown in its equivalent circuit in Fig. 7.1.1, wherein points $\mathrm{A}$ and $\mathrm{B}$ denote the current input terminal, and $\mathrm{C}$ and $\mathrm{D}$ represent the voltage output terminal. $R$ denotes resistance, $L$ represents inductance, and $C$ denotes capacitance. When the input current is taken as reference, the output voltage has a phase shift $\varphi$ :

$$
\varphi \approx \omega L / R-\omega R C .
$$

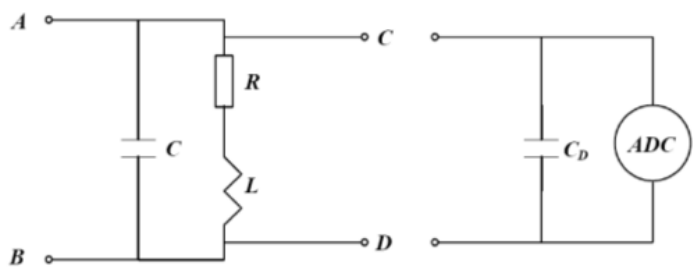

Fig. 7.1.1. Equivalent circuit of shunts.

When an $\mathrm{ADC}$ is used to measure the voltage, its input capacitor $C_{D}$ may generate a new measurement result:

$$
\varphi \approx \omega L / R-\omega R C-\omega R C_{D},
$$

where the function of $C_{D}$ cannot be disregarded. To overcome such influencing factors, a substitution method-based sampling measurement is developed [Wang et al., 2008].

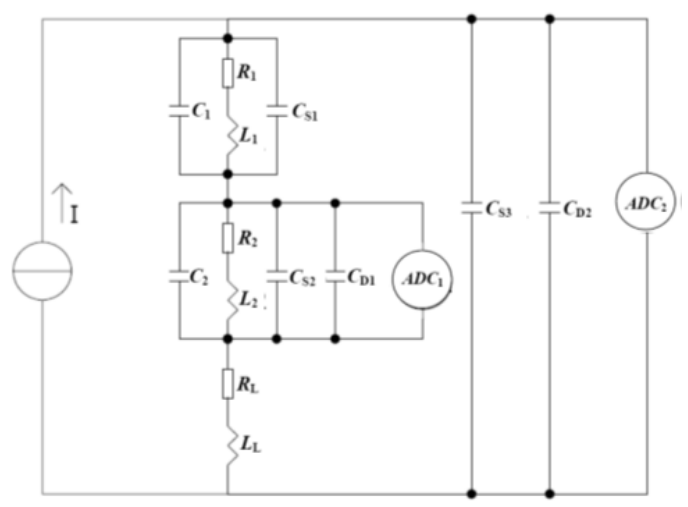

Fig. 7.1.2A. Phase shift measurement step 1. 


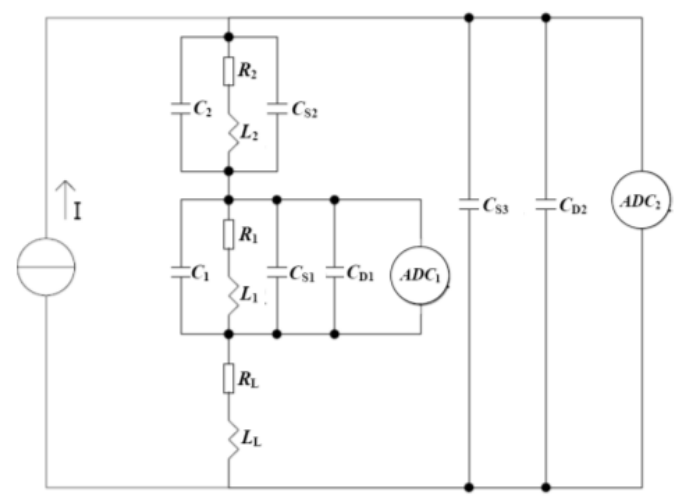

Fig. 7.1.2B. Phase shift measurement step 2.

In Fig.7.1.2A, the shunts, one's phase shift known and another's unknown, are connected in series. Two ADCs simultaneously measure the voltages: one for a shunt near the earth point, and another for the full voltage. The relevant phase difference of $\varphi_{1}-\varphi_{2}$ as the measurement result can be obtained according to Eq. (7.1.2). Fig.7.1.2B shows that the positions of the two shunts are carefully exchanged, and the new phase difference of $\varphi_{3}-\varphi_{4}$ can be obtained.

The influence function of $C_{D 1}$ and $C_{D 2}$ can be eliminated in the difference of both results of $\Delta \varphi=\left(\varphi_{1}-\varphi_{2}\right)-\left(\varphi_{3}-\varphi_{4}\right)$. Suitable wiring may enable $C_{S 1}, C_{S 2}$ to be disregarded, and yields

$$
\Delta \varphi \approx \omega\left(L_{2} / R_{2}-R_{2} C_{2}\right)-\omega\left(L_{1} / R_{1}-R_{1} C_{1}\right) .
$$

On the basis of the phase shift definition of the shunt in Eq. (7.1.5), we infer that the result from Eq. (7.1.7) requires measurement, and then the phase shift of the unknown shunt is determined using the known shunt.

\subsection{Phase shift of voltage dividers}

\subsubsection{Phase shift measurement}

The phase shift of the voltage divider between its output and input, and relative uncertainty is determined in an experiment. Every one of the sets of dividers at the ranges 8, 15, 30, 60, 120,240 , and $500 \mathrm{~V}$ is measured. The experiment is based on a step-up procedure, discussed below.

Step-up Procedure Fig. 7.2.1 depicts the step-up procedure for measuring the phase shift of the dividers.

Preparation: A voltage of $0.8 \mathrm{~V}$ is connected in parallel to both DVMs to eliminate their intrinsic phase difference; that is, the $0^{\circ}$ standard is formulated.

Step 1. A voltage of $0.8 \mathrm{~V}$ is applied parallel to both DVMs (DVM2 at $10 \mathrm{~V}$ and DVM1 at 1 $\mathrm{V})$ without any divider. The phase difference between the 10 and $1 \mathrm{~V}$ range of DVM2 is measured.

Step 2. A voltage of $8 \mathrm{~V}$ is applied parallel to the $8 \mathrm{~V}$ divider (a divider with a range of $8 \mathrm{~V}$ ) and DVM2 in its $10 \mathrm{~V}$ range. The output of the divider (i.e., $0.8 \mathrm{~V}$ ) is connected to 
DVM1 in its $1 \mathrm{~V}$ range. The phase difference between the output and input of the 8 $\mathrm{V}$ divider is measured.

Step 3. The $15 \mathrm{~V}$ divider is measured, and the $8 \mathrm{~V}$ divider is taken as a standard. A voltage of $8 \mathrm{~V}$ is applied parallel to both dividers, and the outputs of both dividers are connected to two DVMs. The phase shift of the $15 \mathrm{~V}$ divider is measured.

Similar procedures are repeated up to the $480 \mathrm{~V}$ divider. The measurement results are shown in Table 7.2.1 and their standard deviations are listed in Table 7.2. 2.

DVM1 DVM2

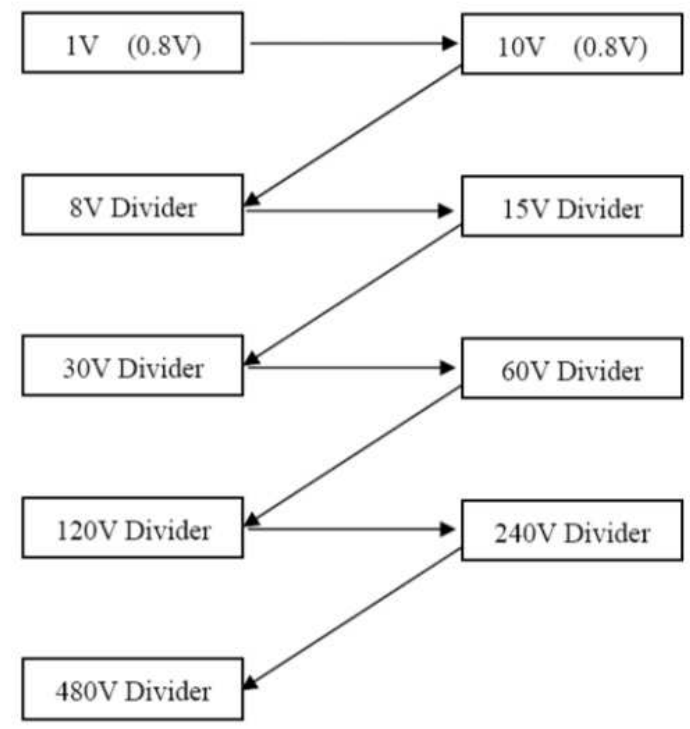

Fig. 7.2.1. Step-up procedure for measuring the phase shift of the voltage dividers.

\begin{tabular}{|c|c|c|c|c|c|c|c|}
\hline Order & 1 & 10 & 20 & 30 & 40 & 50 & 60 \\
\hline $60 \mathrm{~V}$ & 13.0 & 131.9 & 260.3 & 396.1 & 515.4 & 639.5 & 785.9 \\
\hline $120 \mathrm{~V}$ & 14.6 & 143.0 & 282.7 & 430.2 & 559.4 & 694.0 & 851.3 \\
\hline $240 \mathrm{~V}$ & 12.2 & 118.2 & 233.4 & 356.8 & 459.8 & 567.7 & 701.9 \\
\hline $480 \mathrm{~V}$ & -14.4 & -150.8 & -305.7 & -451.1 & -614.9 & -776.7 & -918.4 \\
\hline
\end{tabular}

Table 7.2.1. Test results of phase shift $\varphi_{V k} / \mu \mathrm{rad}$

\begin{tabular}{|c|c|c|c|c|c|c|c|}
\hline Order & 1 & 10 & 20 & 30 & 40 & 50 & 60 \\
\hline $60 \mathrm{~V}$ & 0.0 & 0.5 & 2.7 & 0.6 & 7.1 & 1.1 & 10.3 \\
\hline $120 \mathrm{~V}$ & 0.3 & 4.0 & 5.9 & 10.3 & 12.5 & 14.7 & 20.9 \\
\hline $240 \mathrm{~V}$ & 0.4 & 0.5 & 1.5 & 1.3 & 2.4 & 4.4 & 3.0 \\
\hline $480 \mathrm{~V}$ & 0.6 & 1.4 & 3.7 & 6.1 & 8.7 & 14.2 & 4.7 \\
\hline
\end{tabular}

Table 7.2.2. Standard deviation / $\mu$ rad 


\subsubsection{Uncertainty of phase shift measurement}

The uncertainty of the phase shift is evaluated according to Eq. (7.1.4).

The $0^{\circ}$ standard uncertainty, determined in an experiment, is shown in Table 7.2.3.

\begin{tabular}{|c|c|c|c|c|c|c|c|}
\hline Order & 1 & 10 & 20 & 30 & 40 & 50 & 60 \\
\hline$u(\Delta \varphi)$ & 3.01 & 2.12 & 1.84 & 2.06 & 1.70 & 3.14 & 3.66 \\
\hline
\end{tabular}

Table 7.2.3. Uncertainty of the $0^{\circ}$ standard $/(\mu \mathrm{rad})(k=1)$

Another factor, the half range effect, is considered. A divider obtains the value in its half range but provides the value in its full range to the next divider.

This effect can be measured by comparing two cases: the full and 50\% ranges. The results are shown in Table 7.2.4.

\begin{tabular}{|c|c|c|c|c|c|c|c|}
\hline Order & 1 & 10 & 20 & 30 & 40 & 50 & 60 \\
\hline$\Delta \varphi$ & 2.0 & 2.0 & 3.0 & 4.0 & 5.0 & 6.0 & 6.5 \\
\hline
\end{tabular}

Table 7.2.4. Variation of phase difference between half and full range/( $\mu \mathrm{rad})$

Thus, Table 7.2.5 shows the uncertainty of the phase shift of every divider, including the standard deviation in Table 7.2.2, uncertainty of the $0^{\circ}$ standard in Table 7.2.3, half range effect in Table 7.2.4, and uncertainty of its foregoing divider.

\begin{tabular}{|c|c|c|c|c|c|c|c|}
\hline Order & 1 & 10 & 20 & 30 & 40 & 50 & 60 \\
\hline $60 \mathrm{~V}$ & 6.03 & 5.41 & 7.64 & 9.22 & 11.4 & 13.9 & 15.2 \\
\hline $120 \mathrm{~V}$ & 6.38 & 6.11 & 8.56 & 10.6 & 12.9 & 15.6 & 17.2 \\
\hline $240 \mathrm{~V}$ & 6.71 & 6.47 & 9.16 & 11.3 & 14.0 & 16.9 & 18.5 \\
\hline $480 \mathrm{~V}$ & 7.04 & 6.91 & 10.3 & 13.5 & 17.2 & 22.8 & 20.1 \\
\hline
\end{tabular}

Table 7.2.5. Uncertainty of phase shift of dividers after correction, $u\left(\Delta \varphi_{V k}\right) /(\mu \mathrm{rad})(k=1)$

The $120 \mathrm{~V}$ divider under a $100 \mathrm{~V}$ fundamental has a phase shift of $14.6 \mu \mathrm{rad}$, and its uncertainty is $13 \mu \mathrm{rad}(k=2)$. However, these results can be applied only to the sinusoidal signal and in full range because the experiments are implemented under these conditions.

\subsection{Phase shift of shunts}

A similar step-up procedure (Fig. 7.3.1) is conducted for the shunts from 0.1 to $20 \mathrm{~A}$, and 50 A. An original standard is necessary; that is, a standard resistor of a known time constant developed early at the NIM. With the parameters $R=10 \Omega, \tau=0.03 \times 10^{-9}$, phase shift is less than $6 \times 10^{-7}$ within $3 \mathrm{kHz}$. 


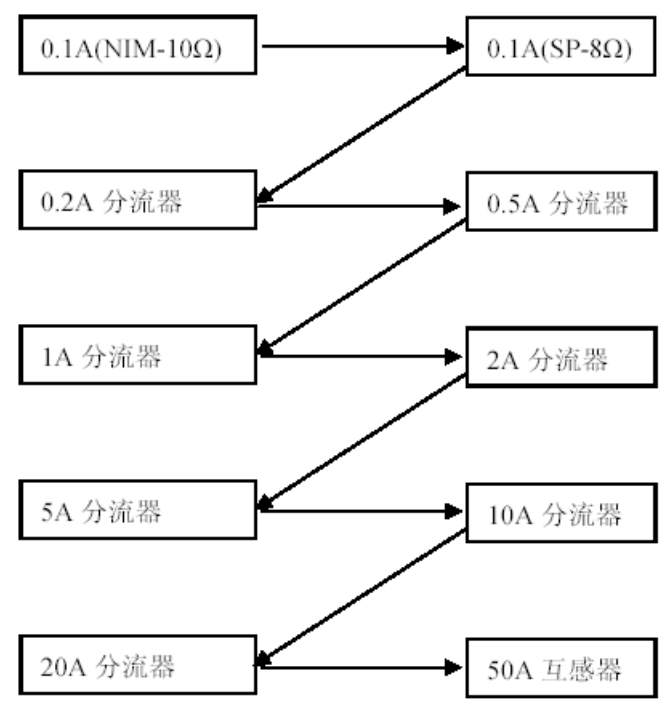

Fig. 7.3.1. Step-up procedure for measuring the phase shift of shunts.
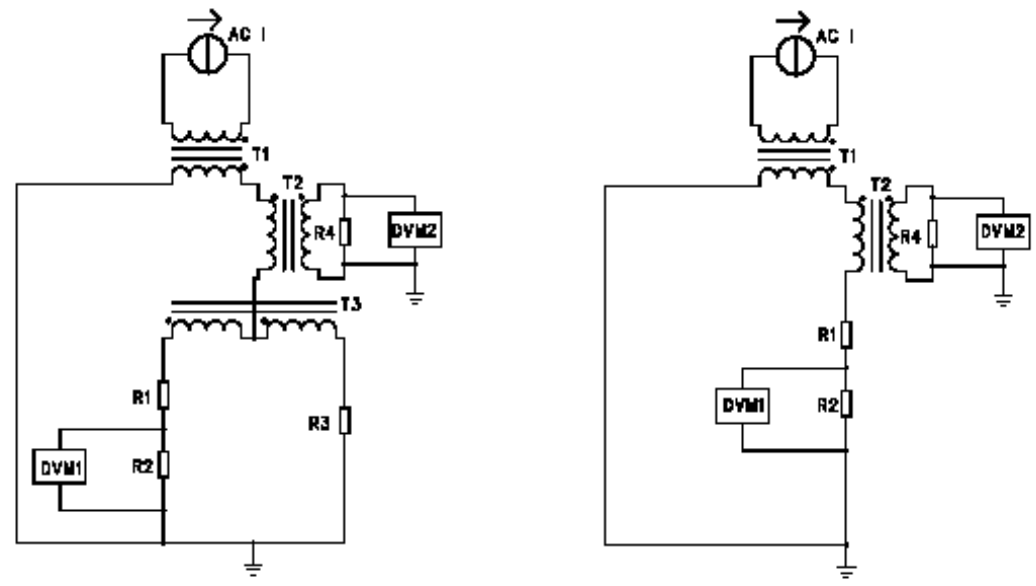

Fig. 7.3.2. Measurement the half range effect of the shunts.

All the shunts and 50 A transformers are measured using the method described in Section 7.1.3. The measurement results for all the shunts are obtained. The results for the shunt of 5 A, including its phase shift and uncertainty, are expressed in Table 7.3.1.

In the uncertainty, all the factors are considered and computed. The half range effect is determined in an experiment, as shown in Fig. 7.3.2. $R_{2}$ is measured initially at the half 
current of $0.5 I$, and then at the full current of $I$. The half range effect can be determined when $R_{4}$ is used as reference.

\begin{tabular}{|c|c|c|c|c|c|c|c|}
\hline Order & 1 & 10 & 20 & 30 & 40 & 50 & 60 \\
\hline$\Delta \varphi_{I k}$ & -1.3 & -3.0 & -0.7 & -.3 & -7.0 & -12.4 & -0.5 \\
\hline$u\left(\Delta \varphi_{I k}\right)$ & 5.99 & 5.38 & 7.64 & 10.1 & 12.4 & 15.2 & 16.9 \\
\hline
\end{tabular}

Table 7.3.1. Phase shift and its uncertainty (after correction) of the 5A shunt, / $\mu \mathrm{rad},(k=1)$

The 5 A shunt under a 5 A fundamental has a phase shift of $-1.3 \mu \mathrm{rad}$, and its uncertainty is $12 \mu \mathrm{rad}(k=2)$. The results can be applied only to the sinusoidal signal and in full range.

\subsection{Conclusion}

The uncertainty evaluation is discussed to measure phase difference by applying DFT to sampling data in NIPS. The step-up procedures are described for the voltage dividers and shunts. The phase shifts and their uncertainties are given. The uncertainty of the phase shift in the fundamental is $13 \mu \mathrm{rad}(k=2)$ for the $120 \mathrm{~V}$ divider, and $12 \mu \mathrm{rad}(k=2)$ for the $5 \mathrm{~A}$ shunt. These specifications fall under the special frequency characteristic. They are discussed further when the results are applied to harmonic power measurement.

\section{Harmonic power measurement}

\subsection{Determination of Harmonic Power}

In the Section 5, DC component $V_{0}$, fundamental component $V_{1}$ and its phase angle $\varphi_{V 1}$, and $k$-th harmonic components $V_{k}$ and their phase angle $\varphi_{V k},(k=2,3, \ldots, w)$ are obtained for a non-sinusoidal voltage signal.

Section 6 details the derivation of DC component $I_{0}$, fundamental component $I_{1}$ and its phase angle $\varphi_{11}, k$-th harmonic components $I_{k}$ and their phase angle $\varphi_{I k}$, and $(k=2,3, \ldots, w)$ for a non-sinusoidal current signal.

From these, we can calculate the harmonic power.

DC power is

$$
P_{0}=V_{0} I_{0} .
$$

Fundamental active power, reactive power, and apparent power are

$$
\begin{gathered}
P_{1}=V_{1} I_{1} \cos \varphi_{1}, \\
Q_{1}=V_{1} I_{1} \sin \varphi_{1}, \\
S_{1}=V_{1} I_{1} .
\end{gathered}
$$

The $k$-th harmonic active power, reactive power, and apparent power are as follows:

$$
P_{k}=V_{k} I_{k} \cos \varphi_{k},
$$




$$
\begin{gathered}
Q_{k}=V_{k} I_{k} \sin \varphi_{k}, \\
S_{k}=V_{k} I_{k},
\end{gathered}
$$

where $\varphi_{1}=\varphi_{V 1}-\varphi_{I 1}, \varphi_{k}=\varphi_{V k}-\varphi_{I k}$, and $k=2,3, \ldots, w$.

\subsection{Uncertainty analysis}

According to the definition in Section 4.1, the fiducial error of harmonic active power can be written as

$$
\delta P_{k}=\Delta P_{k} /\left(V_{1} I_{1}\right)
$$

where $k=1,2, \ldots, w$.

The cases of reactive power and apparent power can be similarly analyzed.

The uncertainty of $\delta P_{k}$, fiducial uncertainty of harmonic active power, $u\left(\Delta P_{k} /\left(V_{1} I_{1}\right)\right)$ in reference to fundamental apparent power has four parts:

$$
u^{2}\left(\Delta P_{k} /\left(V_{1} I_{1}\right)\right)=u_{1}^{2}+u_{2}^{2}+u_{3}^{2}+u_{4}^{2},
$$

where

$$
\begin{gathered}
u_{1}=\left(I_{k} / I_{1}\right) \cos \varphi_{k} u\left(\Delta V_{k} / V_{1}\right), \\
u_{2}=\left(V_{k} / V_{1}\right) \cos \varphi_{k} u\left(\Delta I_{k} / I_{1}\right), \\
u_{3}=\left[\sin \varphi_{k} I_{k} V_{k} /\left(V_{1} I_{1}\right)\right] u\left(\Delta \varphi_{V k}\right), \\
u_{4}=\left[\sin \varphi_{k} I_{k} V_{k} /\left(V_{1} I_{1}\right)\right] u\left(\Delta \varphi_{I k}\right) .
\end{gathered}
$$

Eqs. (8.2.3) and (8.2.4) are dependent on the fiducial uncertainty of the harmonic voltage and current, or $u\left(\Delta V_{k} / V_{1}\right)$ and $u\left(\Delta I_{k} / I_{1}\right)$, respectively. They result from the ratio errors of the dividers and shunts.

Eqs. (8.2.5) and (8.2.6) are dependent on the relative uncertainty of the phase difference of the harmonic voltage and current, or $u\left(\Delta \varphi_{V k}\right)$ and $u\left(\Delta \varphi_{I k}\right)$, respectively. These are analyzed in Section 7. They result from phase angle errors.

When $\varphi_{k}=0^{\circ}$, Eqs. (8.2.5) and (8.2.6) equal zero; when $\varphi_{k}=90^{\circ}$, Eqs. (8.2.3) and (8.2.4) equal zero.

Eq. (8.2.5), $u_{3}$, can be re-written as

$$
u_{3}=\left[\sin \varphi \mathrm{k} \times\left(I_{k} / I_{1}\right)\right]\left[u\left(\Delta \varphi_{V k}\right) \times\left(V_{k} / V_{1}\right)\right] .
$$

where $u\left(\Delta \varphi_{V k}\right)$ is a relative uncertainty of the phase difference of harmonic voltage, which is related to $V_{k}$, and its values in the standard equipment are determined by sampling measurement. The product of $u\left(\Delta \varphi_{V k}\right) \times\left(V_{k} / V_{1}\right)$ indicates that the reference of $u\left(\Delta \varphi_{V k}\right)$ should be converted from $V_{k}$ to $V_{1}$. As a concept of fiducial uncertainty, this product can be called fiducial uncertainty of the phase difference of voltage, denoted as $u\left(\Delta \varphi_{V k} / V_{1}\right)$. Similar to the 
case of voltage (current), we consider the effects of the small component, leakage, noise, and non-linearity.

$$
u\left(\Delta \varphi_{V k} / V_{1}\right)=\left(V_{k} / V_{1}\right) u\left(\Delta \varphi_{V k}\right) .
$$

Considering the non-linearity of amplitude under the harmonic conditions in Section 5.2, the ratio of $\left(V_{k} / V_{1}\right)$ can be converted to $\left[0.8\left(V_{k} / V_{1}\right)+0.2\right]$. With the addition of the resolution component, a new expression is derived:

$$
u^{2}\left(\Delta \varphi_{V k} / V_{1}\right)=\left[0.8\left(V_{k} / V_{1}\right)+0.2\right]^{2} u^{2}\left(\Delta \varphi_{V k}\right)+6.85^{2} .
$$

Thus, Eq. (8.2.5) becomes

$$
u_{3}=\left[\sin \varphi_{k}\left(I_{k} / I_{1}\right)\right] u\left(\Delta \varphi_{V k} / V_{1}\right) .
$$

Eq. (8.2.6), $u_{4}$, can be similarly treated.

$$
\begin{gathered}
u_{4}=\left[\sin \varphi_{k}\left(V_{k} / V_{1}\right)\right] u\left(\Delta \varphi_{I k} I_{1}\right), \\
u^{2}\left(\Delta \varphi_{\mathrm{I} k} / I_{1}\right)=\left[0.7\left(I_{k} / I_{1}\right)+0.3\right]^{2} u^{2}\left(\Delta \varphi_{I k}\right)+7.28^{2} .
\end{gathered}
$$

The digital estimation of $u_{3}$ and $u_{4}$ can be provided.

\subsection{Uncertainty evaluation}

The uncertainty of harmonic active power can be calculated according to Eq. (8.1.5), where parameters $V_{k} / V_{1}, I_{k} / I_{1}$ are taken from the characterizing signal in Table 4.2.1, and uncertainties $u\left(\Delta V_{k} / V_{1}\right), u\left(\Delta I_{k} / I_{1}\right), u\left(\Delta \varphi_{V k}\right)$, and $u\left(\Delta \varphi_{I k}\right)$ are taken from the experimental results in Tables 5.4.1, 6.3.1, 7.2.5, and 7.3.1, respectively. For the cases $\varphi_{k}=0^{\circ}, 30^{\circ}, 60^{\circ}$, and $90^{\circ}$, the calculated results are shown in Table 8.3.1.

\begin{tabular}{|l|l|l|l|l|l|l|l|}
\hline Order & 1 & 10 & 20 & 30 & 40 & 50 & 60 \\
\hline $0^{\circ}$ & 42 & 28 & 4.4 & 3.0 & 1.6 & 0.63 & 0.70 \\
\hline $30^{\circ}$ & 39 & 26 & 4.2 & 2.9 & 1.5 & 0.61 & 0.65 \\
\hline $60^{\circ}$ & 32 & 22 & 3.8 & 2.6 & 1.3 & 0.55 & 0.52 \\
\hline $90^{\circ}$ & 28 & 19 & 3.6 & 2.4 & 1.3 & 0.52 & 0.44 \\
\hline
\end{tabular}

Table 8.3.1. Fiducial uncertainty of active power at $500 \mathrm{~V}, 20 \mathrm{~A}$ under the characterizing signal condition, $U\left(\Delta P_{k} /\left(V_{1} I_{1}\right)\right) /(\mu \mathrm{W} / \mathrm{VA})(k=2)$

In Table 8.3.1 the maximum estimated uncertainty $(k=2)$ is $42 \mu \mathrm{W} / \mathrm{VA}$, which is the uncertainty index of the harmonic active power. At $100 \mathrm{~V}$ and $5 \mathrm{~A}$, however, the maximum estimated uncertainty $(k=2)$ is $36 \mu \mathrm{W} / \mathrm{VA}$.

In practical measurement, in order to obtain the best uncertianty of measurement, for example in the important comparison, the parameters of $V_{k} / V_{1}, I_{k} / I_{1}$ may use of the measuring values instead of the values of the characteristic signal. When the measuring signal consists of only a few harmonics, the resolution component in eqn.(5.4.1), (6.3.1), (8.2.7), (8.2.8) may take smaller values according the practical case. In the NIM's standard 
equipment for the non-sinusoidal signal including 1 to 2 harmonics only, under the best experimental conditions, the value may take 1.00 but not 6.85 or 7.28.

Some factors of interest, such as noise and jitter effects, are already included in the experimental results. For jitter, it exists practically as an uncertainty factor in the time point of sampling. However the author takes the concept that the sampling was at the precise time points still according to the Section 3.1, but, as equivalence, the signal instantaneous amplitudes (the sampling values) were attached additional errors. As far as the aliasing effect is concerned, no analysis can be carried out beyond the $40^{\text {th }}$ harmonic component; the analysis is restricted because the power source of the standard equipment does not provide harmonic component signals with orders higher than the $40^{\text {th }}$ in satisfying the specified maximum harmonic components of the $40^{\text {th }}$ order, as per the IEC standard [IEC, 2002, 2003].

\section{Experimental validation}

The specifications of the standard equipment analyzed and determined in the abovementioned sections are validated in a series of experiments.

\subsection{Orthogonality check}

According to the orthogonality of the trigonometric function, power can be produced when, and only when, voltage and current are at the same frequency. Fundamental voltage and harmonic current do not produce power, a constraint that can be applied to validate the effectiveness of the equipment [Arseneau et al., 1995b, 2001].

Four steps are designed in the experiment to verify the performance of the standard equipment of the NIM. A time division-type power converter with an accuracy of $50 \mathrm{ppm}$ is selected as a reference standard.

Step 1. The input voltage and current signals are fundamental.

Step 2. Voltage is fundamental, and current is a combination of fundamental and a harmonic.

Step 3. Voltage is a combination of fundamental and a harmonic, and current is fundamental.

Step 4. Return to Step 1.

Two sets of non-sinusoidal signals are selected on the basis of (a) IEC61000-3-2 [IEC, 2001] (fundamental voltage, $100 \mathrm{~V}$; current, $3 \mathrm{~A} ; 5^{\text {th }}$ order voltage, $10 \% ; 5^{\text {th }}$ order current, $40 \%$ ), and (b) NRC [Arseneau et al., 1995a, 1997] (voltage, $120 \mathrm{~V}$ with THD =9.5\%; current, $5 \mathrm{~A}$ with $T H D=19.5 \%)$.

With the set of IEC signals at PF $=1.0$, the errors are -1.4 and $-0.7 \mu \mathrm{W} / \mathrm{VA}$ with the $5^{\text {th }}$ order current harmonics and $5^{\text {th }}$ order voltage harmonics included, respectively. With the set of NRC signals, the corresponding errors are -0.6 and $-0.4 \mu \mathrm{W} / \mathrm{VA}$, which can be disregarded.

Two other commercial power meters, one operating under the principle of a thermoconverter and the other under the principle of sampling, are also subjected to the same test. Similar results were obtained for these power meters. 


\subsection{Test on the commercial programmable signal generator}

The commercial programmable signal generator not only provides sinusoidal fundamental waveform voltage and current signals, but also enables the modification of these waveforms to include harmonic components for the calibrations of harmonic meters. It is specified as a $0.02 \%$ device (a standard source).

The performance of the programmable signal generator is also verified using the harmonic power standard equipment. The generator is programmed to produce the set of IEC signals of fundamental $50 \mathrm{~Hz} 100 \mathrm{~V}, 3 \mathrm{~A}$, and $\cos \varphi=1$, and $5^{\text {th }}$ harmonic $10 \mathrm{~V}, 1.2 \mathrm{~A}$, and $\cos \varphi=1$. The measured results are compared with the set values of the signal generator. The fiducial harmonic errors of voltage, current, and power from the DC to the $7^{\text {th }}$ order harmonics are shown in Table 9.2.1 They basically confirm the specifications of the signal generator.

However, a small non-linearity effect in the current output is observed. Larger errors are generated at the DC, 2nd, 4th, and 6th order harmonic currents. This result may be indicative of a non-linearity effect. Given the square of the fundamental component that produces the DC and 2nd order harmonic components, the cross term of the fundamental and 5 th order harmonic component produces the 4 th and 6th harmonic components. A similar but less noticeable phenomenon can also be observed in the voltage output. The occurrence of the non-linearity effect should be confirmed through other tests.

\begin{tabular}{|c|c|c|c|}
\hline Order & Voltage $/(\mu \mathrm{V} / \mathrm{V})$ & Current $/(\mu \mathrm{A} / \mathrm{A})$ & Power $/(\mu \mathrm{W} / \mathrm{VA})$ \\
\hline 0 & 79.5 & $\mathbf{- 2 3 7 . 0}$ & 0.0 \\
\hline 1 & -12.9 & -6.6 & -19.5 \\
\hline 2 & 12.1 & $\mathbf{2 6 . 3}$ & 0.0 \\
\hline 3 & 8.7 & 7.1 & 0.0 \\
\hline 4 & 4.9 & $\mathbf{3 2 . 7}$ & 0.0 \\
\hline 5 & -9.8 & -6.3 & -4.6 \\
\hline 6 & 6.2 & $\mathbf{5 1 . 3}$ & 0.0 \\
\hline 7 & 11.0 & 6.9 & 0.0 \\
\hline
\end{tabular}

Table 9.2.1. Fiducial harmonic errors of the signal generator at the IEC signal

\subsection{Comparison with the national energy standard}

Although the harmonic power standard equipment is designed for harmonic power measurements, it can also be used to measure power/energy under sinusoidal waveform conditions. A comparison against the National Primary Power Standard of the NIM is conducted under test conditions of $100 \mathrm{~V}, 5 \mathrm{~A}, 50 \mathrm{~Hz}, \cos \varphi=1,0.5 \mathrm{lag}$, $0.5 \mathrm{lead}$, zero lag, and zero lead. The errors of the harmonic power standard equipment are no more than -18.6 $\mu \mathrm{W} / \mathrm{VA}$ at all the test conditions. This result is in agreement with previous findings within the evaluated uncertainties of the calibration/experiment.

Bilateral comparisons with the power standards of the National Metrology Institute of Germany PTB and USA NIST are also carried out using a traveling transfer standard under the test conditions $120 \mathrm{~V}, 5 \mathrm{~A}, 50 \mathrm{~Hz}$, power factors of unity, 0.5 lag and lead, and zero lag and lead. The agreement is within $4 \mu \mathrm{W} / \mathrm{VA}$ at all the test points. 


\section{Conclusion}

The harmonic power standard equipment of the NIM is introduced. It is based on the digital sampling technique, which does not require synchronous sampling. It features a special algorithm that compensates for the leakage effect caused by asynchronous sampling. When applied to power measurements with harmonic components of up to the $60^{\text {th }}$ order, computation time is less than 2 seconds even without a large computer memory.

We propose a new concept of uncertainty expression that is related to the fundamental, and define it as the fiducial harmonic uncertainty. This concept is designed to evaluate harmonic measurements. A characterizing waveform signal is reported for the universality of the harmonic uncertainty claim of the NIM standard.

The harmonic power standard operates at fundamental frequencies ranging from 45 to $65 \mathrm{~Hz}$ with harmonic components of up to the $60^{\text {th }}$ order, voltage range from 60 to $500 \mathrm{~V}$, current range from 0.1 to $50 \mathrm{~A}$, and any power factor from zero lag through unity to zero lead. The evaluated uncertainties $(k=2)$ of the harmonic voltages relative to the fundamental voltage, harmonic currents relative to the fundamental current, and harmonic power to the fundamental apparent power are $30 \mu \mathrm{V} / \mathrm{V}, 36 \mu \mathrm{A} / \mathrm{A}$, and $42 \mu \mathrm{VA} / \mathrm{W}$, respectively. These parameters may indicate the function of the principles and the methods described in this chapter, but will not be a limit for the future work.

\section{Acknowledgments}

The author would like to thank Prof. Tang Tongyi (Ph.D. supervisor of the author) of Tsinghua University for his guidance in the investigation of the algorithm, as well as Profs. Zhang Zhonghua and Zhang Deshi of the NIM and Prof. Yang Fusheng of Tsinghua University for their guidance. Appreciation also goes to Mr. Wang Lei of the NIM for his work in the construction of the equipment, as well as Ms. Li Min, Ms. Liu Lijuan, and Mr. Zhou Hao for their contributions to the project. Special thanks to Dr. Eddy So of the National Research Council of Canada for his valuable advice.

\section{References}

Arseneau R., Filipski P. S., and Zelle J. J., (1995a). A portable and stable source of ac voltage current and power. IEEE Trans. Instrum. Meas., Vol.44, (Apr. 1995). pp.433-435, ISSN 0018-9456

Arseneau R. and Filipski P. S., (1995b). A calibration system for evaluation the performance of harmonic power analyzer. IEEE Trans. Power Delivery, Vol.10, (July 1995). pp.1177-1182

Arseneau R., Filipski P.S., and Zelle J.J., (1997). An improved three phase digital recorder system for calibrating power instrumentation. IEEE Trans. Instrum. Meas., Nol. 46, (Apr. 1997). pp.399-402, ISSN 0018-9456

Arseneau R., Sutherland M. E., Zelle J. J., andSvensson S., (2001). Comparison of Nonsinusoidal Calibration Systems at NRC Canada and SP Sweden. IEEE Trans. Instrum. Meas., Vol.50, (Apr. 2001). pp.275-277, ISSN 0018-9456

BIPM, IEC, IFCC, ISO, IUPAC, IUPAP, OIML, (1993a). International Vocabulary of Basic and General Terms in Metrology(Second edition), International Organization for Standardization, ISBN92-67-01075-1, Switzerland

BIPM, IEC, IFCC, ISO, IUPAC, IUPAP, OIML, (1993b). Guide to the Expression of Uncertainty in Measurement, ISBN:92-67-10188-9, Switzerland

Dai X.Z. and Gretsch. R, (1994). Quasi-Synchronous Sampling Algorithm and Its Applications, IEEE Transactions on Instru. \& Meas., Vol.43, No.2, (April 1994). pp.204-209, ISSN 0018-9456 
IEC, International Standard (2001). IEC61000-3-2, Electromagnetic compatibility (EMC)- Part 3-2: Limits- Limits for harmonic current emissions, Switzerland

IEC, International Standard (2002). IEC61000-4-7, Electromagnetic compatibility (EMC)- Part 4-7: Testing and measurement techniques-General guide on harmonics and interharmonics measurements and instrumentation, for power supply systems and equipment connected thereto, Switzerland

IEC, International Standard (2003). 62053-21, Electricity metering equipment (a.c.)Particular requirements, Part 21: Static meters for active energy, Switzerland

Ihlenfeld W.K. et al.,(2003). Evaluation of the synchronous generation and sampling technique. IEEE Trans. Instrum. Meas., Vol., 52, No.2, (2003). pp. 371-374, ISSN 0018-9456

Lu Z.L., (1986). The interpolation and its error analysis for sampling measurement data. Acta Metrologica Sinica, Vol. 7, No. 4, (Oct.1986). pp. 314-322 , ISSN 0018-94561000-1158

Lu Z.L., (1988). An Error Estimate for Quasi-Integer-Period Sampling and an Approach for Improving its Accuracy. IEEE Trans. Instrum. Meas., Vol. 37, No. 2, (June 1988). pp.219-222, ISSN 0018-9456

Lu Z.L., (1991). Study of non-integer-period sampling and development of the standard of power harmonic analyzer, Ph.D. dissertation, Tsinghua Univ., 1991. Available from http://opac.nlc.gov.cn/F/TISYTLKRPTC65TF5JJNT1GG16PLJ6DUXR8CBF5IEKPYPQ 9VPSR-13390?func $=$ full-set-set\&set_number $=301081 \&$ set_entry $=000001 \&$ format $=999$

Lu Z.L., Wang L., and Li M., (2006). Applying DFT of sampling data for determination of phase angle, 2006CPEM Digest, pp.562-563, ISBN:88-7992-228-9, Torino, July 2006

Lu Z.L., Wang L., Li M., Liu L.J., and Zhou H., (2008a). A proposal of definition for uncertainty of harmonic voltage and its experimental determination. 2008CPEM Digest, pp. 446-447, ISBN:978-1-4244-2399-6, Broomfield, CO, Jun. 2008

Lu Z.L., Wang L., Li M., Liu L.J., and Zhou H., (2008b). Uncertainty estimation of phase shift of voltage dividers and shunts measured by sampling approach. 2008CPEM Digest, pp.444-445, ISBN:978-1-4244-2399-6, Broomfield, CO, Jun. 2008

Lu Z.L., Wang L., Li M., Liu L.J., and Zhou H., (2008c). Precision measurement of harmonic current and power at industrial frequency. 2008CPEM Digest, pp.204-205, ISBN:978-1-4244-2399-6, Broomfield, CO, Jun. 2008

Lu Z.L., (2008d). Sampling Strategy for Measurement of Periodic Signals. Electrical Measurement \& Instrumentation, Vol.45, No.514, (Oct.2008). pp.1-6, ISSN 1001-1390

Lu Z.L., Wang L., Li M., Liu L.J., and Zhou H., (2010). Harmonic power standard at NIM and its compensation algorithm. IEEE Trans. Instrum. Meas., Vol. 59, No. 1, (January 2010). pp.180-187, ISSN 0018-9456

Svensson S., (1998). Verification of calibration system for power quality instruments. IEEE Trans. Instrum. Meas., Vol. 47, (Oct. 1998). pp.1391-1394, ISSN 0018-9456

Wang L., Lu Z.L., Li M., Liu L.J., Zhou H., (2008). A New Approach to Determine Phase Shift of Shunt Based on the Sampling Measurement and Substitution Method. CPEM2008 Digest, pp.202-203, ISBN:978-1-4244-2399-6, Broomfield, CO, Jun. 2008

Xue J.H, Yu H.B., Bai J.F., Lu Z.L., (2011). A Novel Algorithm for Harmonic Measurement Based on Multiple-Average, Acta Metrologica Sinica, Vol. 32, No. 5, (Sep.2011). pp.400-406, ISSN 0018-94561000-1158

Zhang J.Q., Zhao X.M. and Sun J.W., (1996). An Automatic Compensation Algorithm of Quasi-Synchronous Sampling for Distorted Power Measurement. IMTC 96 Digest, pp. 951-955, Brussels, June, 1996

Zhang J.T. et al., (2007). A Method on AC Current Measurements Using Binary Inductive Shunts, Euromet EM, Low Frequency Experts Meeting 2007, MIKES, Finland, 2007 


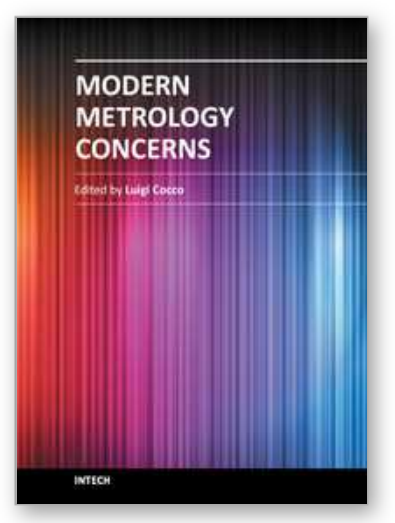

\author{
Modern Metrology Concerns \\ Edited by Dr. Luigi Cocco
}

ISBN 978-953-51-0584-8

Hard cover, 458 pages

Publisher InTech

Published online 16, May, 2012

Published in print edition May, 2012

"What are the recent developments in the field of Metrology?" International leading experts answer this question providing both state of the art presentation and a road map to the future of measurement science. The book is organized in six sections according to the areas of expertise, namely: Introduction; Length, Distance and Surface; Voltage, Current and Frequency; Optics; Time and Relativity; Biology and Medicine. Theoretical basis and applications are explained in accurate and comprehensive manner, providing a valuable reference to researchers and professionals.

\title{
How to reference
}

In order to correctly reference this scholarly work, feel free to copy and paste the following:

Lu Zuliang (2012). Measurement of Harmonic Voltage, Current, and Power at Industrial Frequency, Modern Metrology Concerns, Dr. Luigi Cocco (Ed.), ISBN: 978-953-51-0584-8, InTech, Available from:

$\mathrm{http}: / / \mathrm{www}$.intechopen.com/books/modern-metrology-concerns/measurement-of-harmonic-voltage-currentand-power-at-industrial-frequency

\section{INTECH}

open science | open minds

\section{InTech Europe}

University Campus STeP Ri

Slavka Krautzeka 83/A

51000 Rijeka, Croatia

Phone: +385 (51) 770447

Fax: +385 (51) 686166

www.intechopen.com

\section{InTech China}

Unit 405, Office Block, Hotel Equatorial Shanghai

No.65, Yan An Road (West), Shanghai, 200040, China

中国上海市延安西路65号上海国际贵都大饭店办公楼405单元

Phone: +86-21-62489820

Fax: $+86-21-62489821$ 
(C) 2012 The Author(s). Licensee IntechOpen. This is an open access article distributed under the terms of the Creative Commons Attribution 3.0 License, which permits unrestricted use, distribution, and reproduction in any medium, provided the original work is properly cited. 